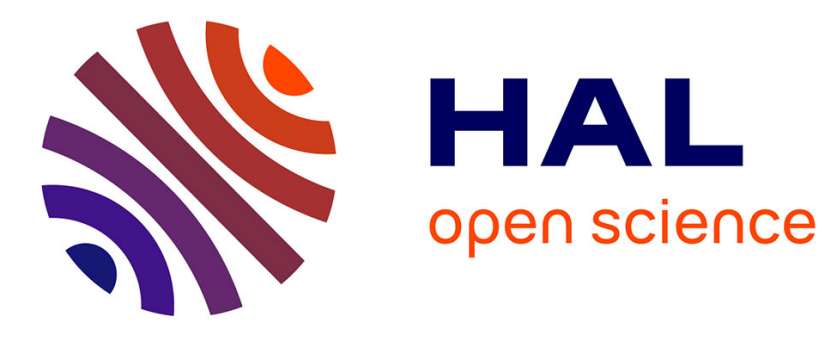

\title{
Internal and inertia-gravity wave focusing at large Stokes numbers
}

\author{
Natalia Shmakova, Bruno Voisin, Joël Sommeria, Jan-Bert Flór
}

\section{To cite this version:}

Natalia Shmakova, Bruno Voisin, Joël Sommeria, Jan-Bert Flór. Internal and inertia-gravity wave focusing at large Stokes numbers. Physical Review Fluids, 2021, 6 (11), pp.114804. 10.1103/PhysRevFluids.6.114804 . hal-03431913

\section{HAL Id: hal-03431913 https://hal.science/hal-03431913}

Submitted on 17 Nov 2021

HAL is a multi-disciplinary open access archive for the deposit and dissemination of scientific research documents, whether they are published or not. The documents may come from teaching and research institutions in France or abroad, or from public or private research centers.
L'archive ouverte pluridisciplinaire HAL, est destinée au dépôt et à la diffusion de documents scientifiques de niveau recherche, publiés ou non, émanant des établissements d'enseignement et de recherche français ou étrangers, des laboratoires publics ou privés. 


\title{
Internal and inertia-gravity wave focusing at large Stokes numbers
}

\author{
Natalia Shmakova $\odot,{ }^{1,2}$ Bruno Voisin $\odot,{ }^{1}$ Joel Sommeria $\odot,{ }^{1}$ and Jan-Bert Flór $\odot^{1, *}$ \\ ${ }^{1}$ Laboratoire des Écoulements Géophysiques et Industriels (LEGI), CNRS, Université Grenoble Alpes, \\ Grenoble INP, 38000 Grenoble, France \\ ${ }^{2}$ Lavrentyev Institute of Hydrodynamics, Siberian Branch of the Russian Academy of Sciences, \\ Prospekt Lavrentyev 15, Novosibirsk 630090, Russia
}

(Received 28 June 2021; accepted 22 October 2021; published 16 November 2021)

In this experimental study we consider the geometric focusing of internal waves by the horizontal oscillation of a torus in a stratified or rotating stratified fluid. In order to minimize viscous dissipation the experiments have been conducted at the 13-m-large Coriolis platform and a large torus was used so that a Stokes number of approximately 6000 was reached. For such large Stokes numbers the waves are bimodal, i.e., they are emitted at the critical locations where the rays are tangential to the torus boundary such that each section of the torus generates for each direction of propagation two wave rays. In contrast to the unimodal case for which these waves diffuse into a single wave beam and there is a single focusing region, there are now four focal regions where the waves emitted from the inner and outer sides of the sections meet. The focusing of the waves on the axis gives rise to strongly nonlinear processes. A linear theory for internal waves emitted by an oscillating torus is presented. For small oscillation amplitudes, we obtain a reasonable agreement of the bimodal wave structure with this linear theory. Resonant wave triads are observed in the stratified (nonrotating) case, similar to the triads of unimodal waves previously observed at moderate Stokes numbers. In the presence of background rotation, no triad is present since the oscillation frequency falls outside the range for which triads can exist. For higher oscillation amplitudes, the waves become highly nonlinear. This results in a spreading of the fundamental wave beams which have relatively less energy than for small oscillation amplitudes, while higher harmonics in the focal region and in the boundary layer of the torus have slightly more energy. A similar tendency is observed for inertia-gravity waves. A large spectrum of waves in resonant interactions is thus observed. This behavior strongly differs from its moderate-Stokes-number counterpart for which higher oscillation amplitudes result in an increase of the energy of the fundamental wave beams. In contrast to observations for small Stokes numbers that showed a decrease in Richardson number with the oscillation amplitude, the Richardson number near the torus and in the focusing region remains constant and close to 1 , and overturning wave motions are absent. The results are discussed in the context of the ocean.

DOI: 10.1103/PhysRevFluids.6.114804

\section{INTRODUCTION}

Tidal currents over the topography at the bottom of the ocean generate internal waves that play an important role for mixing and transport. These internal waves form the so-called internal tides, which through their mixing are estimated to dissipate about $1 \mathrm{TW}$, out of the total tidal energy of about 3 TW [1]. Mixing and transport processes are important for climate modeling. They determine

*jan-bert.flor@legi.cnrs.fr 
the temperature distribution of the ocean and consequently the temperature of its surface, which is in contact with the atmosphere; they also determine the long-term control of the absorption of anthropogenic carbon dioxide and its return to the atmosphere, as well as the general circulation of the ocean (see, e.g., Refs. [2-4]). There is no particular scale at which these processes take place. Energy is continuously exchanged among the different scales of motion with waves and eddies of different sizes interacting in a complex manner. Important processes that contribute to the redistribution of energy among scales are resonant triads and wave streaming [5]. Geometric effects such as wave focusing may favor their occurrence.

In the laboratory, internal waves can be generated by the horizontal oscillation of an object in a stratified fluid at rest. Different objects have been considered, ranging from two-dimensional objects such as cylinders [6,7], continental slopes [8,9], and ridges [10,11] to three-dimensional objects such as spheres [12-14], ellipsoids [15], and seamounts [16]. Recently, more complex objects have been considered that generate converging waves next to diverging waves, the energy of which accumulates in a focal region $[17,18]$.

Wave focusing is a possible scenario for energy concentration in localized zones representing hot spots for incipient overturning in the ocean, far from the region of wave generation. This focusing may occur when the waves reflect on a boundary or bend to a density interface, so that the distance between rays retracts and the energy flux increases. Here we consider geometric focusing that is due to the horizontal curvature of the topography at which the waves are generated, and is therefore three dimensional. In situ measurements confirm the presence of important mixing due to geometric focusing in the Luzon Strait in the South China Sea [19], as well as the Tore Seamount in the West Iberia Margin [20] and the Petite Sole Canyon in the Celtic Sea [21]. For the case of a ringshaped seamount of the Gaussian cross section, the effect of geometric focusing has been analyzed theoretically in [22,23].

The first laboratory experiments on geometric focusing have been conducted with a vertically oscillating torus in a rotating fluid, with the aim of generating a spot of inertial wave turbulence in the focal region with a nonintrusive forcing [24]. In view of the tidal motion in the ocean, wave excitation by horizontal oscillations is however more appropriate. Ermanyuk et al. [17] thus observed that for a horizontally oscillating torus in a linearly stratified fluid the wave amplitude increases toward the focal region. Viscous effects were significant in these experiments. Wave triadic interactions were observed at large oscillation amplitude as well as breaking of the focused waves in the focal region [18]. A nondimensional focusing number was defined that relates the wave amplitude to the maximum Richardson number of the waves, thus allowing for the prediction of overturning motions in the focal region. Those experiments were limited to fairly viscous flows, so that the waves emitted by each cross section of the torus took the form of unimodal beams with maximum wave amplitude at the center of the beam, leading to the formation of two focal regions, one above and one below the torus, as shown schematically in Figs. 1(a) and 1(c).

In contrast to these earlier studies performed in small-scale laboratory experiments, the present study considers internal and inertia-gravity waves for large Stokes numbers so that viscous and dissipative effects are relatively small. The Stokes number is defined as

$$
\mathrm{St}=\omega_{0} a^{2} / v
$$

where $\omega_{0}$ is the oscillation frequency, $a$ the minor radius of the torus, and $v$ the fluid viscosity. In the present experiments, St is of order 6000 for internal waves and 7000 for inertia-gravity waves (compared with $\mathrm{St}=150$ and 260 in [17] and about 250 in [18] and [24]). For such large Stokes numbers, the wave beams are bimodal. The focusing regions obtained in the viscous case mentioned above [see Figs. 1(a) and 1(c)] are now split up into four separate regions of wave focusing, with two focal points on the axis of symmetry and two additional regions in between on either side, as shown in Figs. 1(b) and 1(d). Also, a larger range of scales are generated in the boundary layer of the torus, especially for larger-amplitude forcing, which results in the emission of higher harmonics (see $[25,26])$. 
(a)

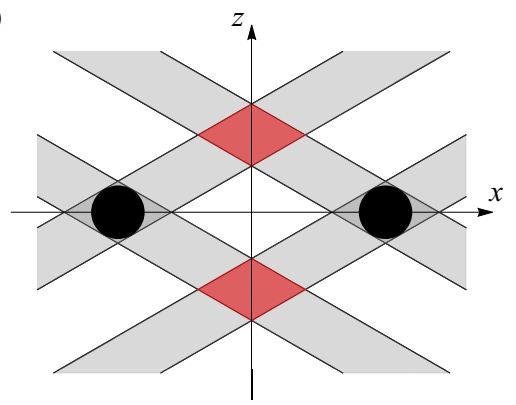

(c)

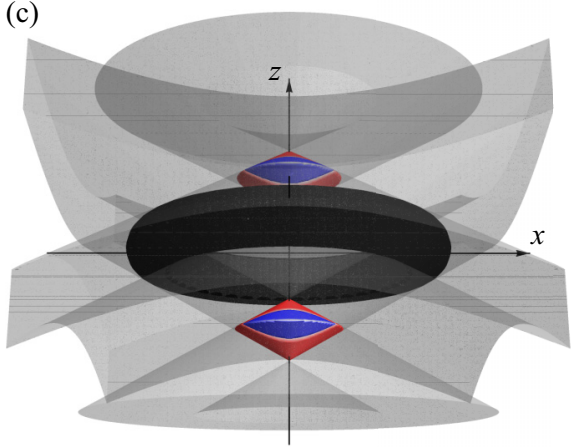

(b)

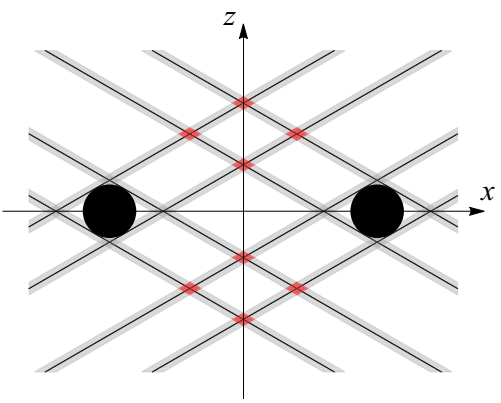

(d)

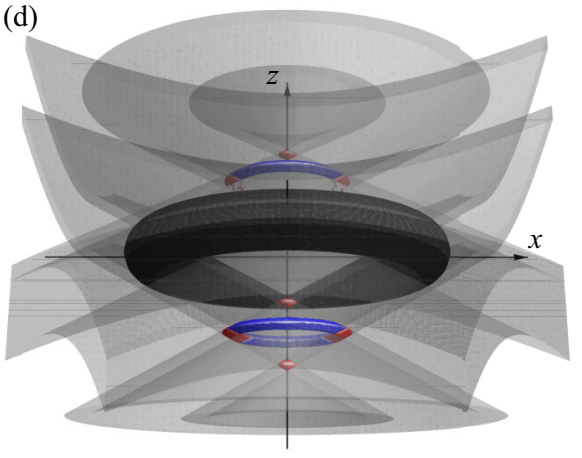

FIG. 1. Focusing geometry for (a), (c) unimodal waves and (b), (d) bimodal waves generated by the horizontal oscillation of a torus of vertical axis, in (a), (b) the vertical plane $x z$ (with $x$ the direction of oscillation and $z$ the torus axis) and (c), (d) perspective view. The torus is shown in black and the critical rays, tangential to it and inclined at a given angle to the vertical determined by the frequency of oscillation, as solid black lines in (a), (b). The wave beams, in gray, are delimited by the critical rays for unimodal waves and are situated around these rays for bimodal waves. Inside their intersections, in blue, the directivity associated with the oscillation of the torus picks up the zones, in the vicinity of the $x z$ plane, where the wave amplitude is most significant, yielding the focal regions in red.

Among the nonlinear processes relevant for the mixing and transport in the ocean, particular attention has been paid to triadic resonant instability, as recently reviewed in [5]. For small oscillation amplitudes, wave triads have also been observed for unimodal waves. For large oscillation amplitudes the flow is abundant in small-scale structures and higher harmonics dominate the flow field. This alters the overall flow structure and trend of the local Richardson number in the focal region.

Below we use for comparison and as a reference field the linear theory presented in [27] for the three-dimensional internal wave field generated by a horizontally oscillating torus, including viscous effects and corrected according to [28]. This reveals the deviation of the experimental results from linearity for large Stokes numbers. The theory is presented in Sec. II and the experimental details, the measurement method, and the data treatment in Sec. III. The experimental results are presented and compared with the linear theory in Sec. IV, followed by a discussion of the triadic resonances. The conclusions in Sec. V discuss these results in the context of recent developments, together with their oceanic applications.

\section{LINEAR THEORY FOR THE NONROTATING CASE}

We consider the horizontal oscillations of a torus of vertical axis, minor radius $a$, and major radius $b$ in a density-stratified fluid of buoyancy frequency $N$. The oscillations, of frequency $\omega_{0}<$ $N$ and amplitude $A$, generate internal waves which propagate away from the torus at the angle 
$\theta=\arccos \left(\omega_{0} / N\right)$ to the vertical, then focus on the axis at the distance $b \cot \theta$ from the center of the torus. Focusing is all the more pronounced when the torus is slender, i.e., $a \ll b$.

A quasi-two-dimensional approach of the problem has been proposed by Ermanyuk et al. [17], applying to internal waves the strip theory of Newman [29] for the motions of a floating slender torus due to incident surface waves. Each section of the torus acts as the section of a horizontal circular cylinder, emitting internal waves calculated by Hurley [30] and Hurley and Keady [31]. The contributions of the two opposite sections in the same azimuthal plane are superposed, then multiplied by a radial factor accounting for energy conservation in the actual cylindrical geometry. The result allows the calculation of the vertical velocity in the focal region but yields infinite horizontal velocity and infinite wave slope on the symmetry axis, thus prohibiting the prediction of incipient overturning and mixing.

We adopt instead a three-dimensional approach built upon the theory of Voisin et al. [13], based on earlier work by Voisin [32], taking the later corrections of [28] into account. An arbitrary oscillating body is represented as a source of mass releasing the volume $\nabla \cdot \mathbf{u}=q e^{-i \omega_{0} t}$ of fluid per unit volume per unit time, with $\mathbf{u}$ the velocity. The body is assumed to have vertical size $2 a$, such that, in a system of Cartesian coordinates $(x, y, z)$ with the $z$-axis vertical, the source satisfies $q(x, y, z)=0$ for $|z|>a$. The velocity components $(u, v, w)$ are given by

$$
\begin{aligned}
(u, v, w)= & \frac{\cos \theta}{8 \pi^{2}} e^{-i \omega_{0} t} \int_{0}^{2 \pi} d \phi_{k}\left[\sin \theta \cos \left(\phi_{k}-\phi\right), \sin \theta \sin \left(\phi_{k}-\phi\right), \cos \theta \operatorname{sgn} z\right] \\
& \times \int_{0}^{\infty} d \kappa \kappa q\left(k_{0}, l_{0}, m_{0}\right) \exp \left(-\beta \kappa^{3}|z| / \cos \theta\right) \exp \left\{i \kappa\left[r_{h} \cos \theta \cos \left(\phi_{k}-\phi\right)-|z| \sin \theta\right]\right\},
\end{aligned}
$$

where $\left(r_{h}, \phi, z\right)$ are the cylindrical polar coordinates,

$$
q(k, l, m)=\iiint q(x, y, z) e^{-i(k x+l y+m z)} d x d y d z
$$

is the source spectrum, taken at the wave vector of components

$$
k_{0}=\kappa \cos \theta \cos \phi_{k}, \quad l_{0}=\kappa \cos \theta \sin \phi_{k}, \quad m_{0}=-\kappa \sin \theta \operatorname{sgn} z+i \beta \kappa^{3} \operatorname{sgn} z / \cos \theta,
$$

and $\beta=v /\left(2 \omega_{0} \tan \theta\right)$ with $v$ the kinematic viscosity. The validity of this result is ascertained for $|z|>a$; for every particular oscillating body, it is expected to hold wherever the integral converges. The analysis stands for low viscosity, namely large Stokes number St $=\omega_{0} a^{2} / v$ (5800 in the present experiments), and small amplitude of oscillation, namely small Keulegan-Carpenter number $\mathrm{Ke}=$ $A / a$ (between $1 / 6$ and 1 in the present experiments). Previous experiments with the sphere [13,14] suggest that such linear analysis is quantitatively valid for Ke $\lesssim 0.2$, and remains qualitatively valid for $0.2 \lesssim \mathrm{Ke} \lesssim 0.6$, say.

As for Ermanyuk et al. [17], the representation of the torus is based on its slenderness, namely on the assumption that the aspect ratio $\epsilon=b / a$ ( 5 in the present experiments) is large. A summary derivation has been given in [27]. Each section of the torus behaves as the section of a horizontal circular cylinder, with representation given in [28]. Using the wide separation between the local scale $a$ of a single section and the global scale $b$ of the whole torus we obtain the spectrum

$$
q(k, l, m)=-4 i \pi^{2} N a b A e^{i(\theta-\Theta)} J_{0}\left(b \sqrt{k^{2}+m^{2}}\right) J_{1}\left(a \sqrt{k^{2}+l^{2}+m^{2}}\right) k / \sqrt{k^{2}+l^{2}+m^{2}},
$$

where $J_{n}$ denotes the Bessel function of order $n$; in this spectrum, the factor $J_{1}\left(a \sqrt{k^{2}+l^{2}+m^{2}}\right) k / \sqrt{k^{2}+l^{2}+m^{2}}$ represents the effect of an individual section and the factor $J_{0}\left(b \sqrt{k^{2}+m^{2}}\right)$ the interference between all the sections. The origin of the system of coordinates has been taken at the position of the center of the torus at rest, the $x$ axis along its direction of oscillation, and the initial phase $\Theta$ has been chosen such that the position of the center at $t$ is $x=A \sin \left(\omega_{0} t+\Theta\right)$. In the present experiments the time origin $t=0$ is taken at the instant when the torus changes direction at the rightmost point of its oscillation, so that $\Theta=\pi / 2$. 
The fluid velocity follows as

$$
\begin{aligned}
U= & i \pi \epsilon \cos \theta \sin \theta e^{-i\left(\omega_{0} t+\Theta-\theta\right)} \int_{0}^{\infty} K J_{0}(K \epsilon \cos \theta) J_{1}\left(K-i \frac{K^{3}}{2 \mathrm{St}}\right) \exp \left(-\frac{K^{3}|Z|}{2 \mathrm{St} \sin \theta}\right) \\
& \times\left[J_{2}\left(K R_{h} \cos \theta\right) \frac{X^{2}}{R_{h}^{2}}-\frac{J_{1}\left(K R_{h} \cos \theta\right)}{K R_{h} \cos \theta}\right] \exp (-i K|Z| \sin \theta) d K, \\
V= & i \pi \epsilon \cos \theta \sin \theta e^{-i\left(\omega_{0} t+\Theta-\theta\right)} \int_{0}^{\infty} K J_{0}(K \epsilon \cos \theta) J_{1}\left(K-i \frac{K^{3}}{2 \mathrm{St}}\right) \exp \left(-\frac{K^{3}|Z|}{2 \mathrm{St} \sin \theta}\right) \\
& \times J_{2}\left(K R_{h} \cos \theta\right) \frac{X Y}{R_{h}^{2}} \exp (-i K|Z| \sin \theta) d K, \\
W= & \pi \epsilon \cos ^{2} \theta e^{-i\left(\omega_{0} t+\Theta-\theta\right)} \operatorname{sgn} Z \int_{0}^{\infty} K J_{0}(K \epsilon \cos \theta) J_{1}\left(K-i \frac{K^{3}}{2 \mathrm{St}}\right) \exp \left(-\frac{K^{3}|Z|}{2 \mathrm{St} \sin \theta}\right) \\
& \times J_{1}\left(K R_{h} \cos \theta\right) \frac{X}{R_{h}} \exp (-i K|Z| \sin \theta) d K,
\end{aligned}
$$

where the position has been normalized as $(X, Y, Z)=(x, y, z) / a$, with $R_{h}=\sqrt{X^{2}+Y^{2}}$, and the velocity as $(U, V, W)=(u, v, w) /\left(\omega_{0} A\right)$. This formula is valid for $|Z|>\sin \theta$, namely above and below the critical levels $Z= \pm \sin \theta$ at which the wave rays are tangential to the torus on either side of the horizontal midplane $Z=0$.

In the present experiments the Stokes number $\mathrm{St}=5800$ is very high. At such high values, the waves close to the torus are only significant at the critical rays, forming in each azimuthal plane two St Andrew's crosses tangential to each section of the torus above and below, as shown in Fig. 1(b). The wave beams formed in this way are called bimodal, since the wave profile across each beam contains two peaks, one per St Andrew's cross. As the waves propagate away from the torus the rays thicken owing to viscosity, their width varying as the 1/3 power of the propagation distance. Ultimately the rays merge, yielding a unimodal profile with a single peak at the center of the beam. The transition, described in greater detail in [13], takes place at a distance from the torus of order $a \mathrm{St} / 50$, that is about $100 a$ here, far outside the experimental domain.

This picture of the wave field assumes that a steady state has been reached. Before that, at finite time $t$ after the impulsive start-up of the motion at $t=0$, the velocity remains given by (6) but the upper limit of integration is replaced by $K=\omega_{0} t \sin \theta /|Z|$.

\section{EXPERIMENTAL SETUP AND MEASUREMENTS}

\section{A. Coriolis platform and wave generator}

The experiments have been conducted at the Coriolis rotating platform in Grenoble. The total diameter of the platform is $16 \mathrm{~m}$, the tank diameter $13 \mathrm{~m}$, and the maximum fluid height $1 \mathrm{~m}$. The platform is designed for the experimental modeling of geophysical processes in the presence of background rotation and/or density stratification. Its large scale allows the study of ocean dynamics in the presence of weak viscous effects.

To generate the waves, a torus cut in half horizontally as shown in Fig. 2(b) was fixed on a plexiglass horizontal plate which oscillated horizontally at the surface of the fluid [see Fig. 2(a)]. In this manner, the fluid depth was used optimally. This is because the free surface of a stratified fluid is approximately rigid for internal waves (see, e.g., Sec. 10.5 in [33]). When we suppose in addition that the flow at the plate is free slip, we may expect by symmetry considerations the same wave field as predicted by the theory for a full torus. The torus had major radius $b=75 \mathrm{~cm}$ and minor radius $a=15 \mathrm{~cm}$. It oscillated horizontally at the center of the tank with frequency $\omega_{0}$ and amplitude $A$ in the range of values $A=2.5,5,10$, and $15 \mathrm{~cm}$. 
(a)

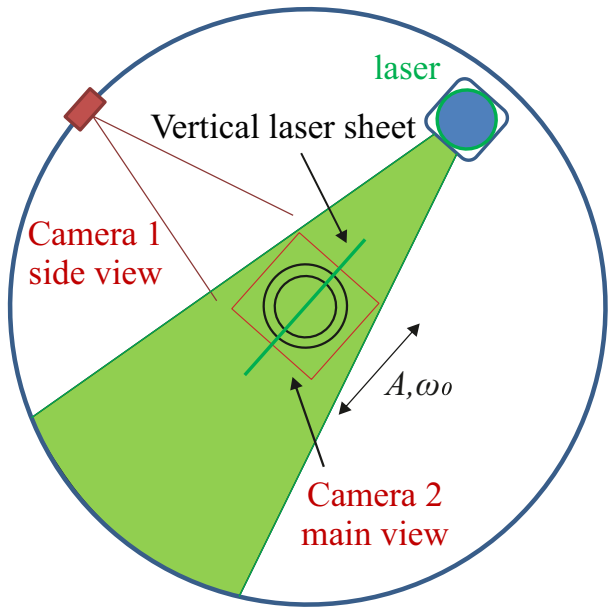

(b)

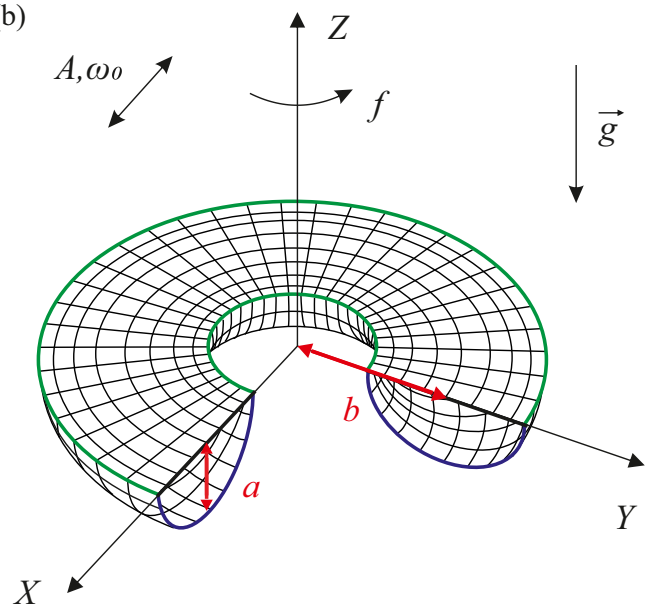

FIG. 2. Schematic sketch of the experiments at the Coriolis platform: (a) top view of the setup showing the $3 \mathrm{~m} \times 3 \mathrm{~m}$ view area of the camera (main view) and (b) parameters of the torus used as a wave generator. The torus is of minor radius $a=15 \mathrm{~cm}$ and major radius $b=75 \mathrm{~cm}$, and oscillates in the $x$ direction with amplitude $A$ and frequency $\omega_{0}$.

The tank was filled to a depth $H=90 \mathrm{~cm}$ with a linearly stratified fluid of constant buoyancy frequency $N=0.5 \mathrm{~s}^{-1}$ for all experiments. The stratification was measured with two conductivity probes before the experiment at a position close to the torus. A third conductivity probe was placed close to the center of the focal zone to measure the waves and turbulence there. For the study of inertia-gravity waves the tank rotated at a rate of $0.1 \mathrm{rad} / \mathrm{s}$ yielding a Coriolis parameter $f=$ $0.2 \mathrm{~s}^{-1}$, so that the Prandtl ratio was $f / N=0.4$.

In all experiments, the oscillation frequency $\omega_{0}$ was chosen so that the focal region had its center $z=-b \cot \theta$ at mid-depth $z=-H / 2$ and the angle of propagation to the vertical was $\theta=\arctan (2 b / H)=59^{\circ}$. Given the dispersion relation

$$
\omega_{0}=\sqrt{N^{2} \cos ^{2} \theta+f^{2} \sin ^{2} \theta}
$$

this implied a frequency ratio $\omega_{0} / N=0.51$ for internal waves and $\omega_{0} / N=0.62$ for inertia-gravity waves. With a kinematic viscosity $v=0.01 \mathrm{~cm}^{2} / \mathrm{s}$ the Stokes number St defined in Eq. (1) was equal to 5800 for internal waves and 7000 for inertia-gravity waves. Viscous effects were thus relatively small compared to most existing laboratory experiments.

The wave amplitude is commonly characterized by the Keulegan-Carpenter number, $\mathrm{Ke}=A / a$, which ranged from $1 / 6$ to 1 in our experiments. This number characterizes the amplitude of the waves close to the torus so that for small values of Ke the waves are linear. To characterize the amplitude of the waves in the focal zone, Shmakova and Flór [18] introduced an alternative number, Fo, given by

$$
\text { Fo }=\operatorname{Ke}(b / a)^{-1 / 2} \sin ^{1 / 2} \theta \cos \theta,
$$

where $\operatorname{Ke}(b / a)^{-1 / 2}=A^{\prime} / a$ is the geometric focusing factor, with $A^{\prime}=A(b / a)^{-1 / 2}$ the amplitude of oscillation of the torus required to obtain wave amplitude $A$ in the focal region, and $\sin ^{1 / 2} \theta \cos \theta$ represents the variation in wave amplitude with $\theta$. Wave breaking was observed in moderate-St flows for Fo $\approx 0.24$. The experimental parameters are summarized in Table I.

For reference, a Cartesian coordinate system has been introduced with its origin at the center of the torus at rest. The horizontal $x$ and $y$ axes are parallel and perpendicular to the oscillation 
TABLE I. Experimental parameters at the Coriolis platform.

\begin{tabular}{lcccccc}
\hline \hline & PIV & $\omega_{0} / N$ & $N\left(\mathrm{~s}^{-1}\right)$ & $f\left(\mathrm{~s}^{-1}\right)$ & $\mathrm{Ke}$ & Fo \\
\hline Expt. 1.1 & PIV & 0.51 & 0.5 & 0 & $0.17 ; 0.33 ; 0.67 ; 1.0$ & $0.036 ; 0.071 ; 0.140 ; 0.210$ \\
Expt. 1.2 & Volume PIV & 0.51 & 0.5 & 0 & 0.17 & 0.036 \\
Expt. 2.1 & PIV & 0.62 & 0.5 & 0.2 & $0.17 ; 0.33 ; 0.67 ; 1.0$ & $0.036 ; 0.071 ; 0.140 ; 0.210$ \\
Expt. 2.2 & Volume PIV & 0.62 & 0.5 & 0.2 & 0.17 & 0.036 \\
\hline \hline
\end{tabular}

direction, respectively, and the $z$ axis points upward perpendicular to the surface of the fluid [see Fig. 2(b)]. The coordinates have been normalized with the minor radius $a$, as $(X, Y, Z)=(x, y, z) / a$.

\section{B. Wave visualization and data treatment}

The flow was measured using standard two-dimensional particle image velocimetry (PIV), or volume PIV. About 5 liters of $200 \mu \mathrm{m}$ polystyrene particles, and in the stratified rotating case of smaller $60 \mu \mathrm{m} \mathrm{Orgasol}{ }^{\circledR}$ particles, were seeded in the neighborhood of the torus just before the experiment. Depending on the choice of PIV technique, the laser sheet generated with a $6 \mathrm{~W}$ Spectra-Physics laser was positioned either horizontally or vertically. The vertical sheet was fixed parallel to the direction of oscillation and illuminated particles in the center plane of the torus $(Y=0)$, and images were taken with a frequency of $10 \mathrm{~Hz}$ from the side with a 12-bit Dalsa camera with $1024 \times 1024$ pixel resolution [see Fig. 2(a)].

Volume PIV measurements required a specific setup and specific settings of the laser and camera. A high-speed Falcon camera with a $2432 \times 1728$ pixel resolution recorded the particle motion around the torus from the top of which a view is shown in Fig. 2(a). The laser sheet was positioned horizontally and was driven vertically by a computer-controlled motor, synchronized with the camera recordings by in-house software designed with LABVIEW. The sheet moved over a total distance of typically $35 \pm 3 \mathrm{~cm}$ around the zone of wave focusing. The time interval between images in one volume was about $20 \mathrm{~ms}$ and the time interval between two volumes, say a volume pair, was a maximum of $1 \mathrm{~s}$. The velocity vectors were calculated for each volume pair as explained below, and the time interval between two volume pairs was set at $3 \mathrm{~s}$ to ensure the return of the laser sheet to its initial position.

The data were analyzed with the UVMAT/CIVX program developed at LEGI [34]. Each volume pair consisted of pairs of images taken at the same level. The horizontal velocity field with components $u$ and $v$ was calculated from the correlation of these image pairs. The vertical velocity $w$ was then calculated by integrating the continuity equation over the distance between two images in a volume. The velocity components obtained from two- and three-dimensional PIV measurements were normalized as $(U, V, W)=(u, v, w) /\left(\omega_{0} A\right)$.

All the data were analyzed in the steady regime as obtained after 12 oscillation periods during which the wave field built up from a state of rest (see, e.g., the spectra later in Fig. 12). An example of this buildup at a position close to the lower focal point is given in Fig. 3, illustrating how, in the focal region, the steady state is reached after 15 oscillation periods and a reasonably good approximation of this state after say 12 periods.

\section{EXPERIMENTAL RESULTS}

\section{A. Observations and comparison with linear theory}

\section{Internal gravity waves}

Figure 4 shows the vertical view of the steady internal wave field in the $X Z$ plane, for the horizontal velocity $U$, the vertical velocity $W$, and the scaled transverse vorticity $\xi_{y} / N$ with $\xi_{y}=\partial u / \partial z-\partial w / \partial x$. The top row represents the field predicted by the linear theory. One notices 

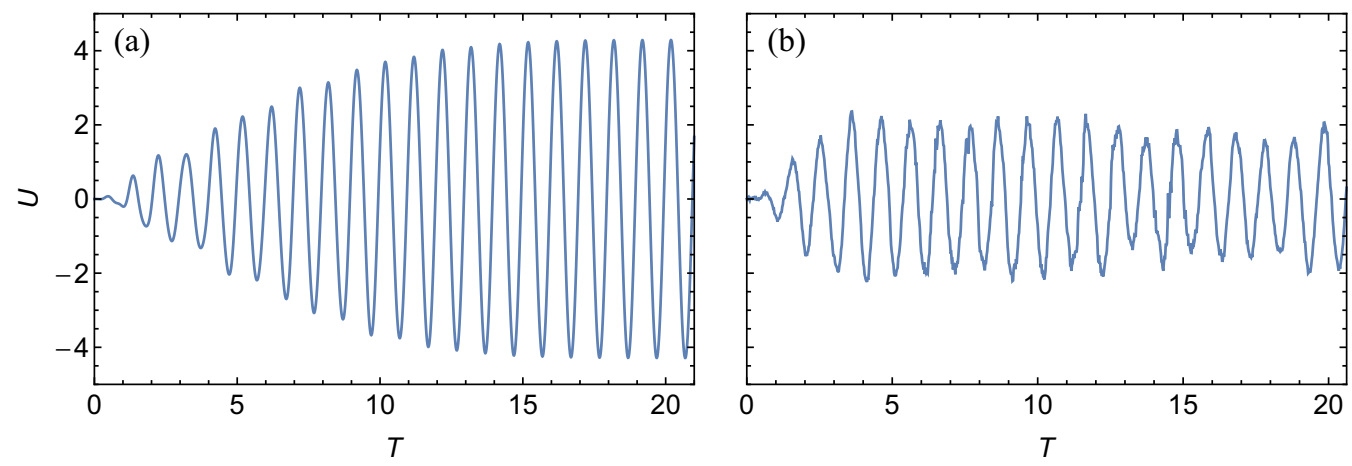

FIG. 3. Evolution of the horizontal velocity $U$ with time $T=\omega_{0} t /(2 \pi)$ since the start-up, for internal waves at the lower focal point on the symmetry axis $x=y=0$ : (a) theory at the predicted position $z=-b \cot \theta-a \csc \theta \approx-62 \mathrm{~cm}$ of the focus and (b) experiment at its measured position $z=-52 \mathrm{~cm}$, for $\mathrm{Ke}=0.17$, corresponding to Expt. 1.1 from Table I.

the bimodal structure of the wave beams, with the two focal points on the axis of the torus and the two additional points in between on either side, as anticipated in Fig. 1(b). The foci show up in Figs. 4(a)-4(c) as four corners of a lozenge-shaped region at the center of the image. For these bimodal waves, the phases at the left and right sides of a section of the torus are identical for the horizontal velocity and opposite for the vertical velocity, since there is a pressure high at the front of the moving section pushing fluid backwards up and down and a pressure low at its back sucking fluid in forwards from above and from below. Depending on their position in the focal region, the interacting waves have opposite or equal phases [see Fig. 4(c)]. The experimental results are discussed below for increasing oscillation amplitude. For comparison with those obtained for moderate Stokes numbers we refer to Ermanyuk et al. [17] and Shmakova and Flór [18].

For small oscillation amplitudes, $\mathrm{Ke}=0.17$ (and $\mathrm{Fo}=0.036$ ), we observe a bimodal structure similar to the theory [see Figs. 4(d)-4(f)], except for one important difference: the downwardpropagating rays emitted at the critical points on the outer sides of the torus are indeed present, but the upward-propagating rays emitted on the inner sides and reflected at the supporting plate are absent. Instead, we see downward-propagating rays coming from the corner regions at the intersection between the torus and the plate, with less energy. The boundary layer at the plate certainly plays a role in this phenomenon, but the exact mechanism is unclear. Theoretically, for the edgewise oscillations of a horizontal disk [35], there is no discernible laminar boundary layer at such high Stokes number. Experimentally, for the horizontal oscillations of a vertical knife edge [10], the use of a sliding stage extending over the bottom of the tank does not prevent the formation of a bimodal wave structure, combining a direct ray and a reflected ray at the stage (see especially their Fig. 6). Accordingly, the present observation is specific to the boundary layer at the plate in our setup, a layer both turbulent and constrained by the torus forming, at the scale of the layer, an impenetrable vertical wall along its circumference.

The boundary layer at the torus, of Reynolds number $\mathrm{Re}=U a / v=\mathrm{Ke} \mathrm{St}=960$ in (d)-(f), 1900 in (g)-(i), 3900 in (j)-(l), and 5800 in (m)-(o), is also turbulent and populated with small-scale motion, resulting in diffuse and thick wave rays, and inefficient wave generation such that the wave amplitude is smaller than expected. Also more energy is distributed to higher harmonics [15,25], discussed further in the following and best noticeable in plots of the vertical velocity, showing almost vertical wave beams emitted from the torus [see Fig. 4(e)]. All three effects (ray thickening, decrease of the fundamental wave, and increase of the higher harmonics) become more pronounced as Ke increases. By contrast, experiments for moderate Stokes numbers, e.g., near an oscillating sphere (see [13]), show that the two rays of each bimodal wave beam are sharp, and the wave amplitude at small $\mathrm{Ke}$ is close to its linear prediction. One may therefore expect that the wave 

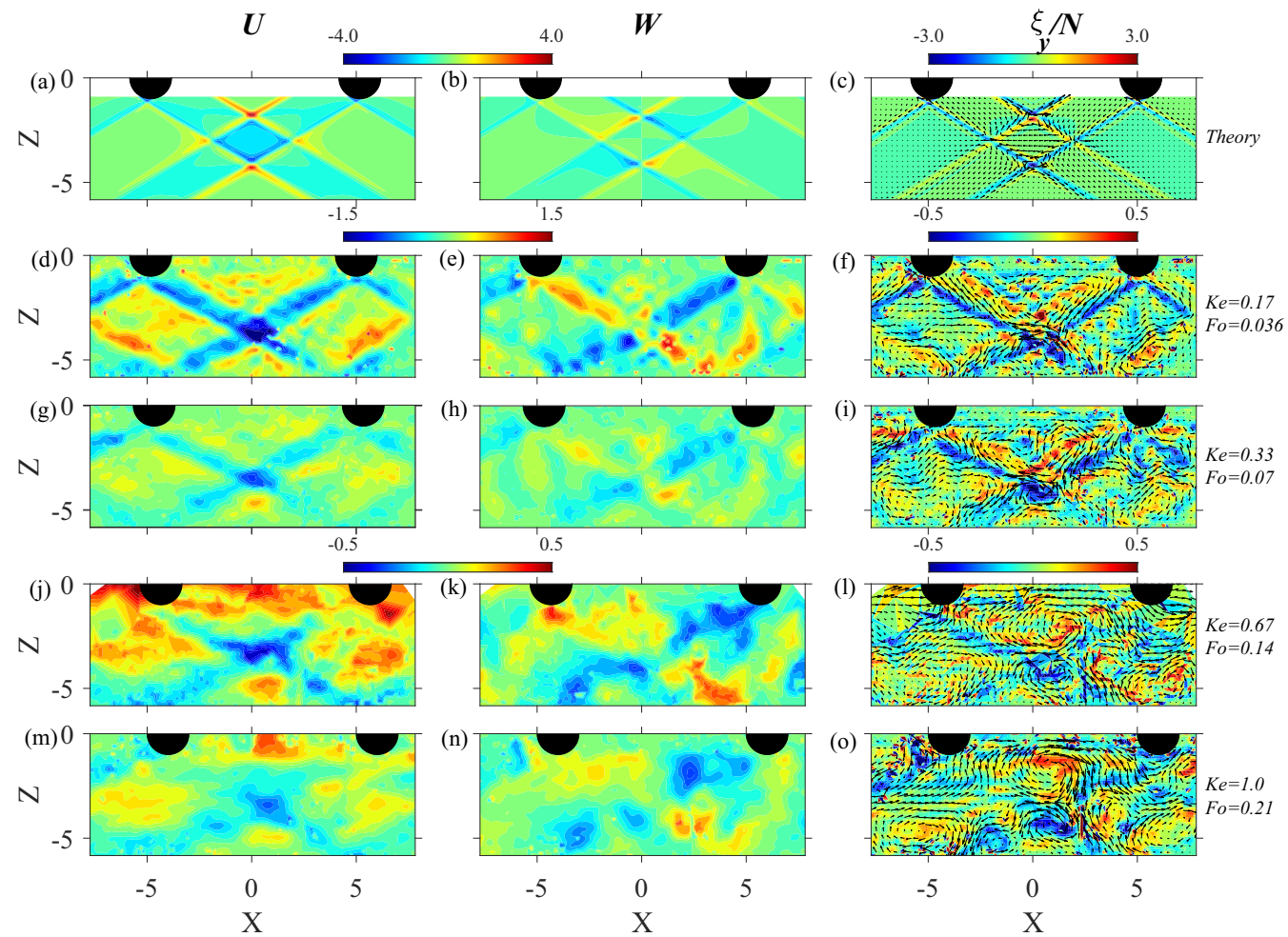

FIG. 4. Instantaneous fields of internal gravity waves at phase $\omega_{0} t=0$ of the 17 th oscillation period in the vertical plane $Y=0$ for the horizontal velocity $U$ (left column), vertical velocity $W$ (middle column), and transverse vorticity $\xi_{y} / N$ with velocity vectors (right column). The theoretical predictions (6) are shown in the first row, together with the experimental results at $\mathrm{Ke}=0.17,0.33,0.67$, and 1.0 in the following rows, respectively. The color scales apply to the images situated below them. See Expt. 1.1 from Table I.

rays will also be sharp for moderate Stokes numbers for an oscillating torus, in agreement with the theoretical prediction shown in Figs. 4(a)-4(c). As discussed earlier in Sec. II, only at a large distance that is out of the region of interest would the rays merge into a unimodal wave beam. (A detailed comparison of the experimental results at moderate Stokes numbers with the linear theory will be shown elsewhere.)

Scrutinizing Figs. 4(d) and 4(f) reveals that the wave beams have sometimes an oscillatory pattern along their length; this pattern is typical of triadic resonant instability and has also been observed in experiments at moderate Stokes numbers [see Figs. 3(e) and 3(g) in [17]]. With increasing oscillation amplitude, $\mathrm{Ke}>0.17$, the fundamental wave decreases in amplitude [see Figs. 4(g), 4(j), 4(m), and 4(h), 4(k), 4(n)]. As will be shown quantitatively in Sec. IV B, the higher harmonics generated in the boundary layer and in the focal region increase in amplitude. As a result, the wave pattern exhibits a different structure compared to that at low oscillation amplitudes.

The instantaneous vorticity and velocity fields in Figs. 4(f), 4(i), 4(1), and 4(o) reveal the breaking up of straight rays into small vortical motions near the torus and the focal region, appearing as horizontally elongated patches of high shear [see Figs. 4(f) and 4(i)] similar as in layerwise stratified turbulence. However, their oscillation in sign over a single wave period reveals nonlinear wave motions and excludes long-living horizontal structures as one would expect in layerwise stratified turbulence (see Supplemental Material I [36]).

A close-up of the phase-averaged first harmonic is shown in Fig. 5 for the horizontal velocity. As mentioned above, apart from the thickening of the wave rays emitted at the torus boundary, and the 


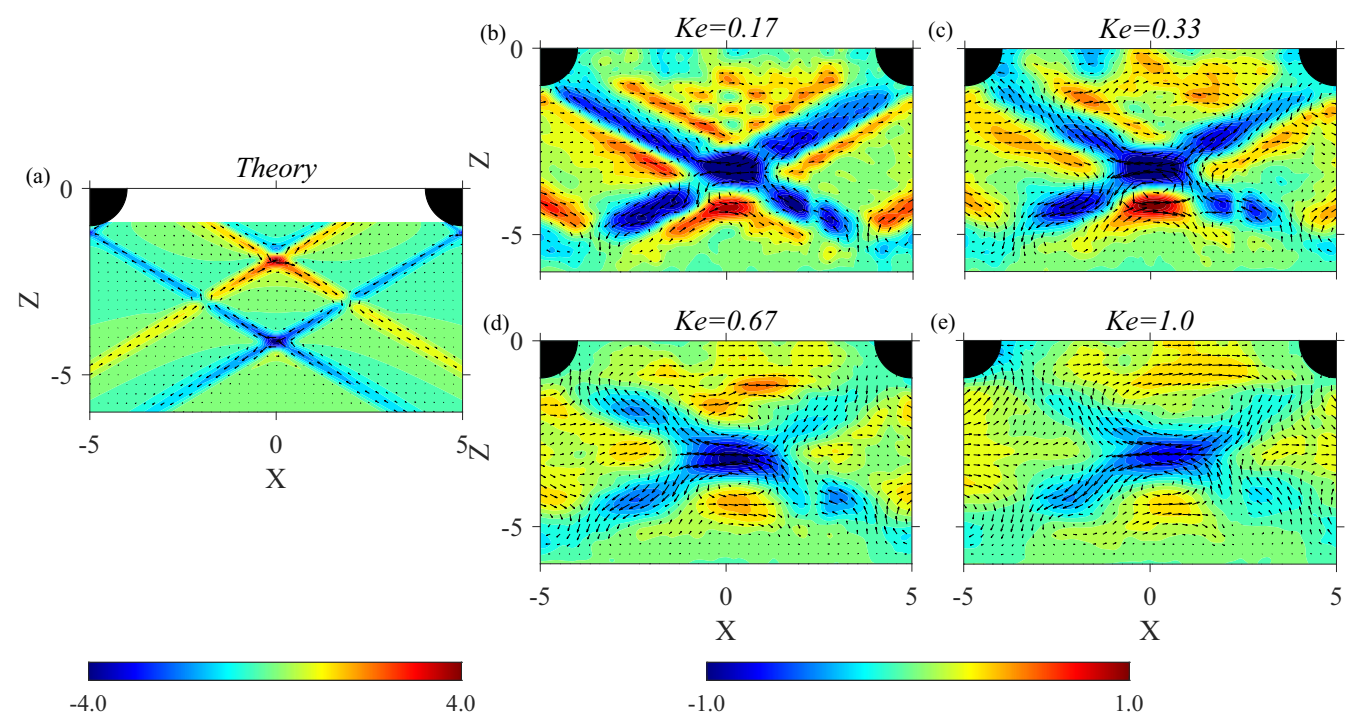

FIG. 5. Close-up of the phase-averaged horizontal velocity $U$ (color) and velocity vectors (arrows) of internal gravity waves at phase $\omega_{0} t=0$ of the 17th oscillation period in the vertical plane $Y=0$ comparing (a) the theoretical predictions (6) and (b)-(e) the experimental results for $\mathrm{Ke}=0.17,0.33,0.67$, and 1.0, respectively. See Expt. 1.1 from Table I.

displacement of the reflected rays to an origin at the corner intersections between the torus and its supporting plate, the theory predicts the lozengelike shape of the focal region fairly well. For higher $\mathrm{Ke}$, the first harmonic becomes diffuse and its amplitude gradually decreases.

Since the nondimensional oscillation frequency is $\omega_{0} / N=0.51$, all the harmonics but the first should be evanescent. However, filtering the velocity field at frequency $2 \omega_{0}$ (again based on phase averaging) reveals the propagating second harmonic, generated close to the torus (Fig. 6). These
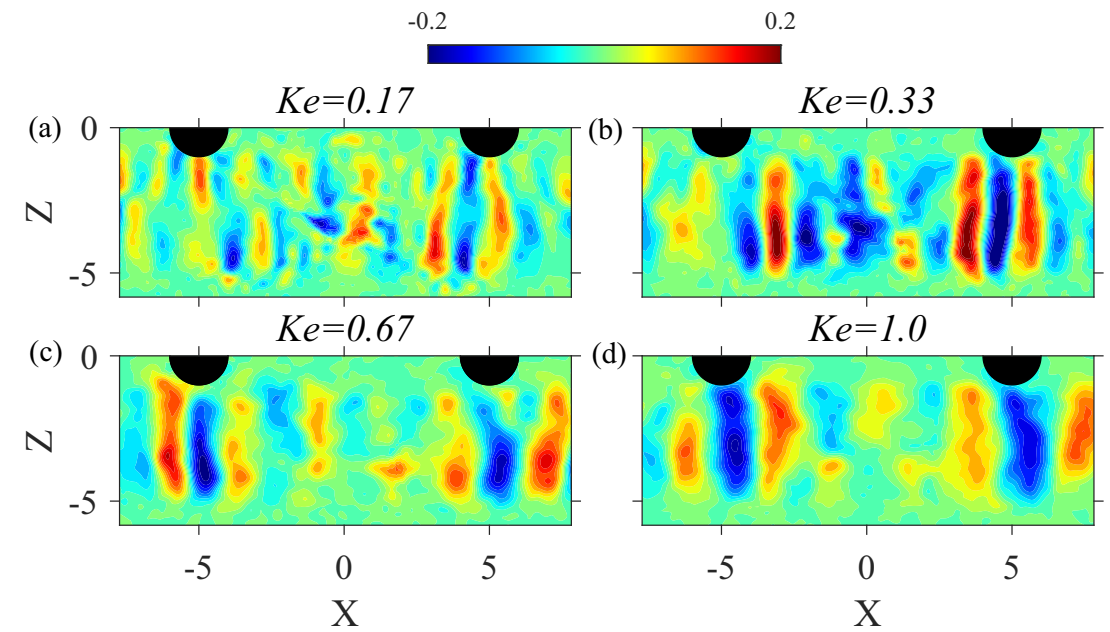

FIG. 6. Experimental patterns of the instantaneous vertical velocity $W$ of internal gravity waves filtered at frequency $\omega=2 \omega_{0}$, at phase $\omega_{0} t=0$ of the 17 th oscillation period in the vertical plane $Y=0$ with (a) Ke $=$ 0.17 , (b) $\mathrm{Ke}=0.33$, (c) $\mathrm{Ke}=0.67$, and (d) $\mathrm{Ke}=1.0$. See Expt. 1.1 from Table I. 

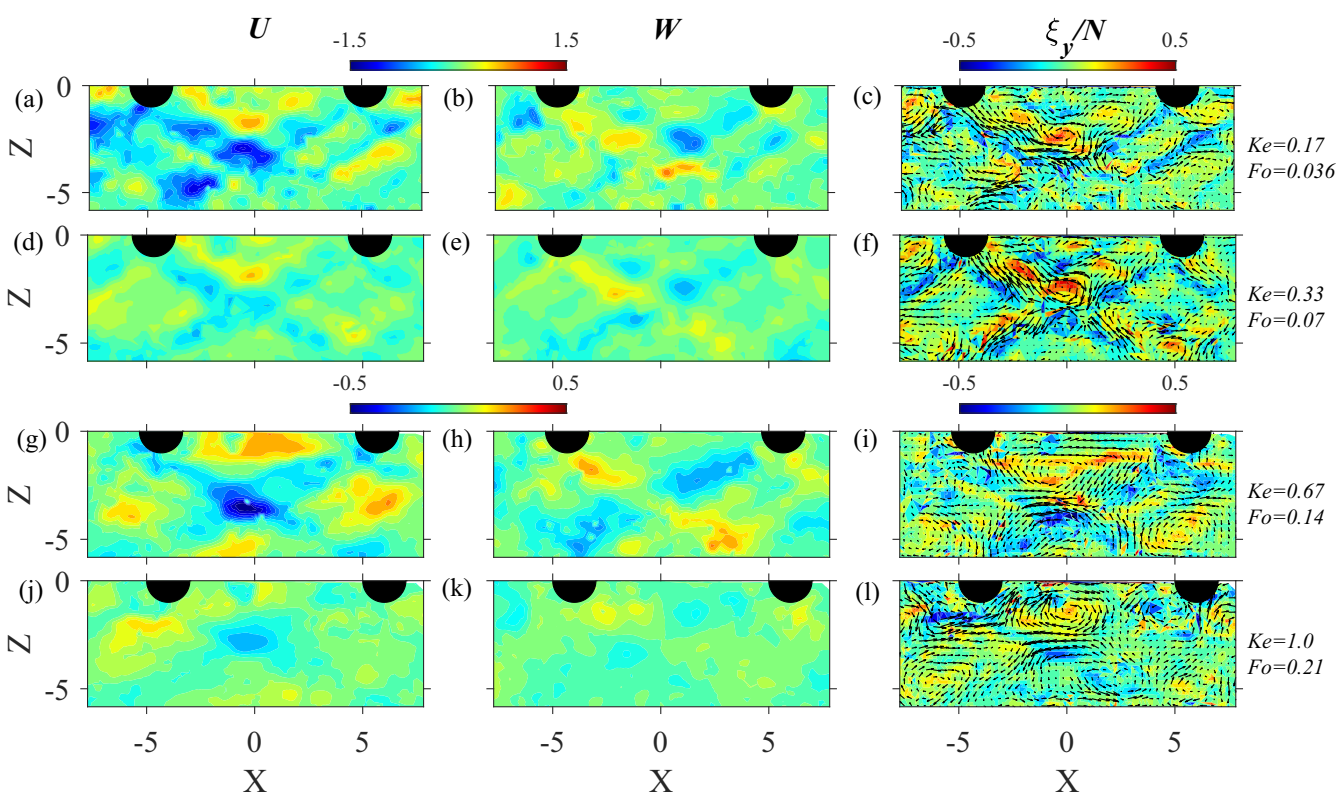

(k)
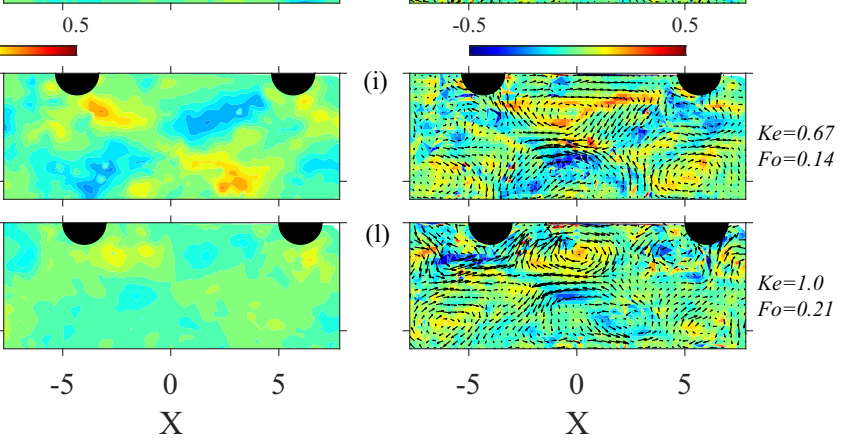

FIG. 7. Same as Fig. 4 for inertia-gravity waves, measured in the 15 th oscillation period. See Expt. 2.1 from Table I.

second harmonic waves are nearly vertical and thus their horizontal velocity component has the same order as the experimental noise, while the vertical velocity component is well pronounced.

For inertial waves, Nosan et al. [37] have shown theoretically and experimentally how, in a rotating fluid, waves of frequency slightly larger than the Coriolis parameter $f$ may propagate horizontally over large distances, limited only by viscosity, despite being vertically evanescent. They mentioned the same phenomenon in a stratified fluid, where internal waves of frequency slightly larger than the buoyancy frequency $N$ are evanescent horizontally but may propagate vertically over large distances, limited only by viscosity. In the present low-viscosity experiments, this may explain the presence of a vertically propagating second harmonic of frequency $2 \omega_{0}=1.02 \mathrm{~N}$.

\section{Inertia-gravity waves}

The evolution of inertia-gravity waves with increasing Ke is shown in Fig. 7. Even for small forcing amplitude, the wave beams break up into small patches with intense motion also outside the wave beams. Along a vertical line through the focal region the vorticity alternates in sign [see Fig. 7(f)]. Correspondingly, the vertical and horizontal velocity change sign in about the same region. This difference in vertical distribution of vorticity is even more clear when comparing Fig. 7(f) with Fig. 4(i) for internal waves. This difference is most likely due to the presence of background vorticity that is stretched along the vertical, so that there is higher vorticity outside the wave region.

With increasing $\mathrm{Ke}$, one notices a number of significant changes. The vertical mode that is apparent from the number of maxima and minima along a vertical line in Fig. 7 decreases from a predominant mode 4 at $\mathrm{Ke}=0.17$ to a predominant mode 2 at $\mathrm{Ke}=1$, as is especially visible in Fig. 8 representing the phase-averaged first harmonic. The structure of this mode becomes diffuse and, although less obvious than in the nonrotating case shown above, a second harmonic component is present and increases in amplitude with Ke. The frequency of this component is $2 \omega_{0}=1.24 \mathrm{~N}$, too far above $N$ for the mechanism present in Fig. 6 to play a role, so that it is unlikely that the second harmonic component is made of waves. Indeed, there is mainly noise at frequency 


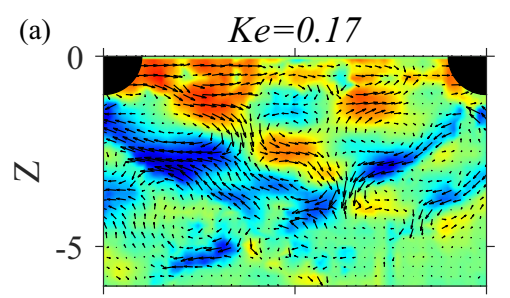

(b)

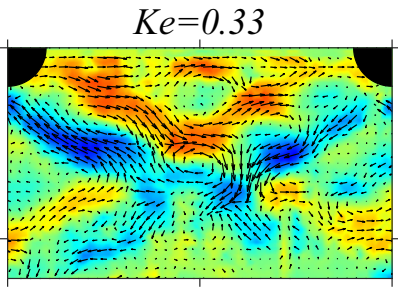

(c)

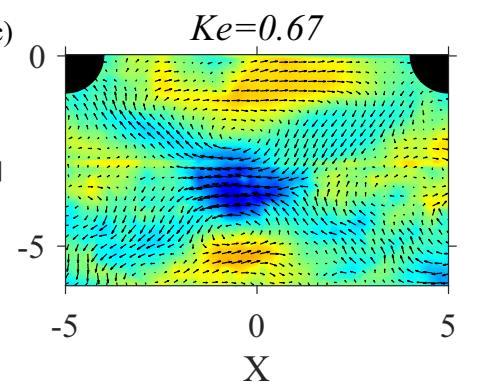

(d)

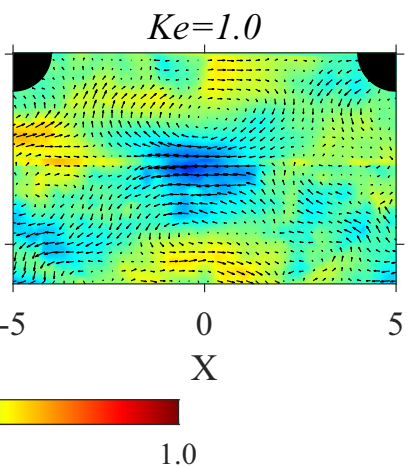

FIG. 8. Same as Fig. 5 for inertia-gravity waves, showing the experimental results measured in the 15 th oscillation period. See Expt. 2.1 from Table I.

$2 \omega_{0}$ for low Ke numbers, and the associated counterpart of Fig. 6 is therefore not shown. The vertical and also horizontal wave amplitudes of internal as well as inertia-gravity waves decrease significantly, leading to a dominant presence of higher harmonics, which is in sharp contrast to the moderate-Stokes-number case discussed in [17] and Fig. 4(b) of [18] at Fo $=0.16$. Further, it is noteworthy that the amplitude of the inertia-gravity waves is 1.5 times larger than that of internal waves.

\section{Richardson number and wave overturning}

The Richardson number Ri is determined from the local shear and mean stratification (see [18]) as

$$
\mathrm{Ri}=\frac{N^{2}}{(\partial u / \partial z)^{2}}=\frac{N^{2} / \omega_{0}^{2}}{(\partial U / \partial Z)^{2}} \approx \frac{N^{2}}{(\Delta u / \Delta z)^{2}},
$$

where the stratification $N$ is measured prior to the experiment and the velocity variation $\Delta u$ is measured from absolute values of the PIV data in the lower focal region on the axis over a grid distance of $\Delta z=12$ pixels corresponding to about $3 \mathrm{~cm}$. The values of $\Delta u$ were averaged over two oscillation periods and their standard deviation was used as an estimate of the error of these measurements. Figure 9 combines the results for the moderate-Stokes-number laminar flow of [18] with those for the present high-Stokes-number flow. For moderate Stokes numbers, Ri clearly decreases with oscillation amplitude, represented by Fo. But for high Stokes numbers, Ri is about constant and around 1 for internal waves, and even increases for inertia-gravity waves. These trends are in coherence with the above observations of a decreasing wave amplitude with increasing oscillation amplitude for large Stokes numbers.

The observation of a constant Richardson number larger than 1 for different forcing amplitudes implies that no overturning (wave breaking) occurs. Indeed, nonlinear oscillations are observed from the particle motions in the experiments (see the vorticity in Figs. 4 and 7), but they never reach a point of breaking. On the other hand, we note that more energy is present in higher modes, an effect that is even more remarkable for higher forcing amplitudes (see Fig. 14 below). These effects are 


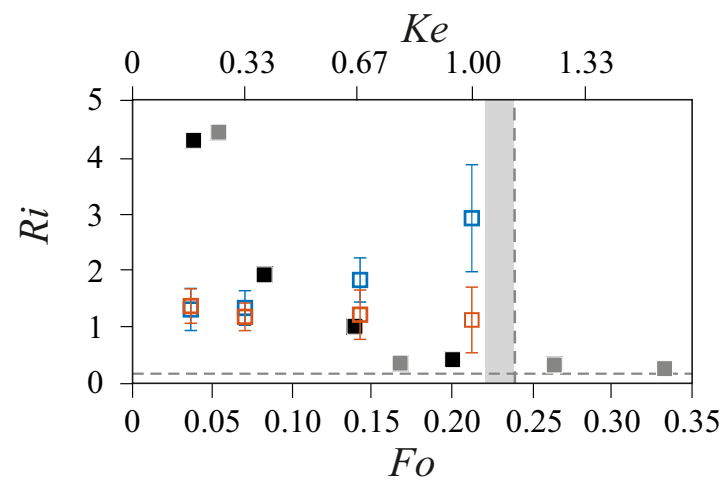

FIG. 9. Variations of the Richardson number Ri with the focusing number Fo and the Keulegan-Carpenter number Ke at $\epsilon=5$, combining the PIV and LIF data of [18] (black and gray symbols) at moderate Stokes numbers St $=270$ and 230, respectively, with the present PIV data in the focal zone of Expts. 1.1 for internal waves (red symbols) and 2.1 for inertia-gravity waves (blue symbols) at high Stokes number St $=5800$ and 7000 , respectively. The error bars represent the standard deviation of the measurements.

stronger for inertia-gravity waves, leading to higher values of the Richardson number in coherence with the decrease in fundamental wave compared to the higher harmonic waves. This would suggest that, for the large Stokes numbers in the oceanic boundary layer, the Richardson number will also be rather $O(1)$, thus excluding overturning motions of topographically induced internal waves.

\section{Comparison between internal and inertia-gravity waves}

For comparison Fig. 10 shows the patterns of vertical vorticity $\xi_{z} / N$, with $\xi_{z}=\partial v / \partial x-\partial u / \partial y$, and horizontal divergence $(\partial u / \partial x+\partial v / \partial y) / N$ for the top views obtained with the theory for internal waves [Figs. 10(a) and 10(b)], the observation of internal waves [Figs. 10(c) and 10(d)], and the observation of inertia-gravity waves [Figs. 10(e) and 10(f)]. The observations were done for the smallest oscillation amplitude, $\mathrm{Ke}=0.17$. According to the linear theory, there is no vertical vorticity in the horizontal plane and the oscillation due to the wave motion causes a streamline pattern in the focal region that resembles a source-sink flow [see Figs. 10(a) and 10(b)] corresponding qualitatively to the experimental observation in Figs. 10(c) and 10(d). The dipolar vorticity pattern in the middle of the focal zone [see Fig. 10(c)] is generated by nonlinear effects and is therefore absent in the linear approach [see Fig. 10(a)]. Half a wave cycle later, the experimental data show that the vorticity pattern is alternated, with the dipole oriented in the opposite direction.

In the presence of background rotation, the vertical motion represented by the divergence in Fig. 10(f) causes the stretching and squeezing of the background vorticity, leading to positive vorticity in the regions with upward vertical motion and negative vorticity in the regions with downward vertical motion [see Fig. 10(e)]. Due to the presence of this vorticity in the regions of nonzero divergence, the flow changes into a Yin-Yang-like structure [Fig. 10(e)]. Taking the average of the vertical vorticity over a single period, there is a small remaining motion of about $0.3 \%$ of the phase-averaged vorticity in both cases, the stratified case as well as the stratified rotating case. This average was taken after 20 periods, indicating that the deposit of momentum due to nonlinear effects is small.

The three-dimensional view of the vertical vorticity field is reconstructed from the volume PIV measurements, and its instantaneous isosurfaces are presented in Figs. 11(a) and 11(b) for internal and inertia-gravity waves, respectively. In both cases, the vorticity is blinking from positive to negative, and vice versa, every half period (see Supplemental Material II [36]). The dipolar vorticity pattern in the nonrotating case [Fig. 11(a)] is only confined to the focal region, suggesting nonlinear effects occurring only in the focal zone. For inertia-gravity waves [Fig. 11(b)], this 

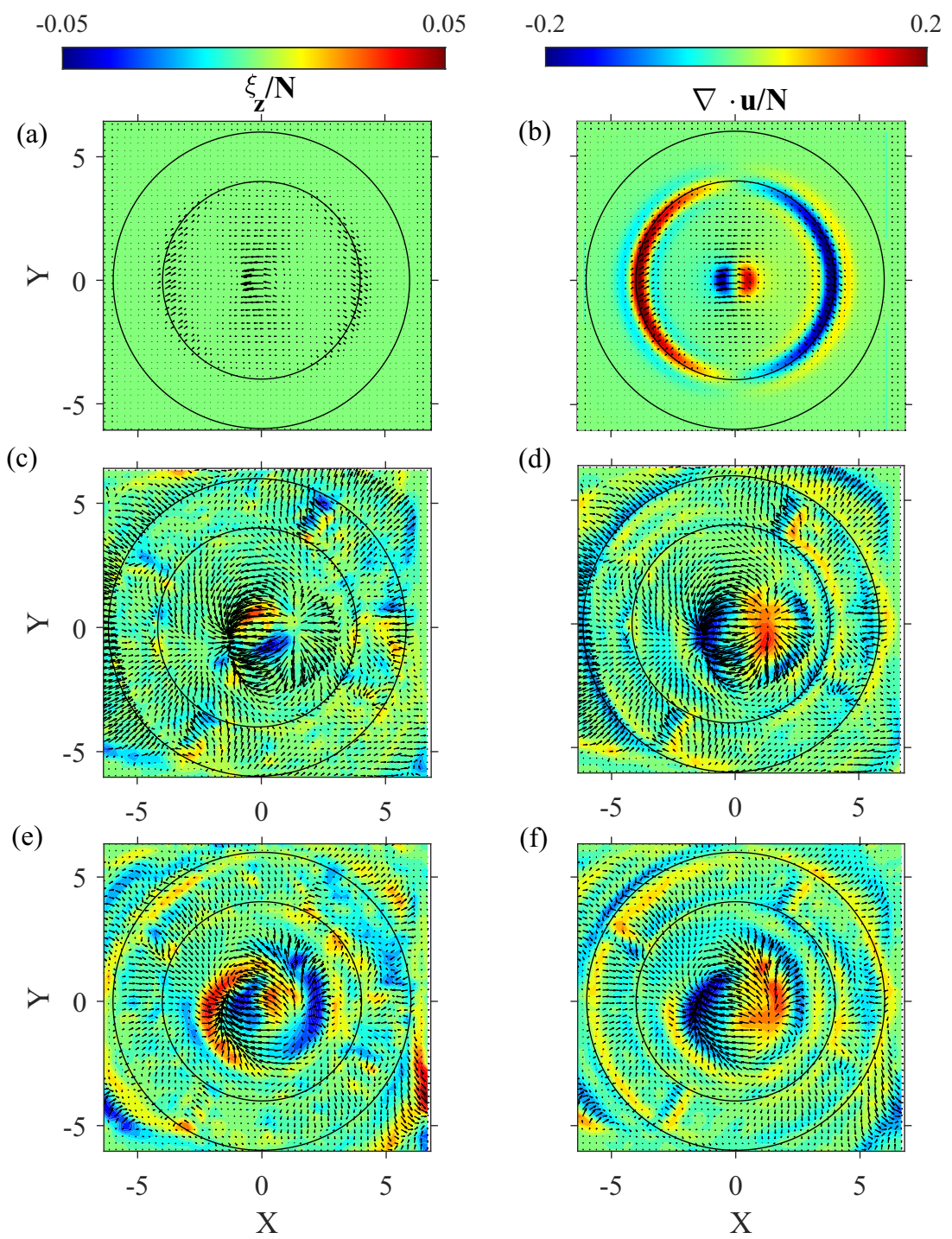

FIG. 10. Top view of the fundamental wave of the instantaneous vertical vorticity field (left column) and horizontal divergence (right column) at phase $\omega_{0} t=\pi$ of the 23rd oscillation period in the horizontal plane $Z=-4.2$ (i.e., at the lower focal point), together with horizontal velocity vectors. The theoretical calculations of internal waves in (a), (b) are followed with the experimental determinations of the first harmonic waves, filtered with the Hilbert transform [38], for (c), (d) internal waves and (e), (f) inertia-gravity waves at Ke = 0.17. See Expts. 1.2 and 2.2 from Table I.

structure extends from the surface to the bottom, suggesting nonlinear effects over the entire depth. Due to the presence of background motion the positive and negative vorticity regions are twisted around each other [Fig. 11(b)], giving rise to the Ying-Yang structure mentioned above. In the case of inertia-gravity waves, this motion takes place over two-thirds of the depth. (Videos of the three-dimensional vorticity field of the internal wave field are shown in Supplemental Material II [36]). The Rossby radius of deformation was calculated as $R_{0}=(N d) / f$, with $d=2 H / 3$ the vertical extent of the structure and $H=90 \mathrm{~cm}$ the fluid depth, and predicts the size of the vortex 
(a)

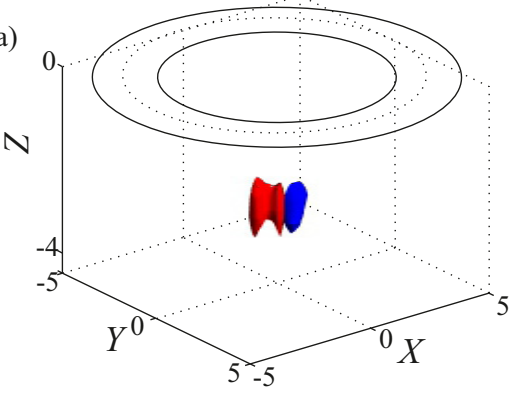

(b)

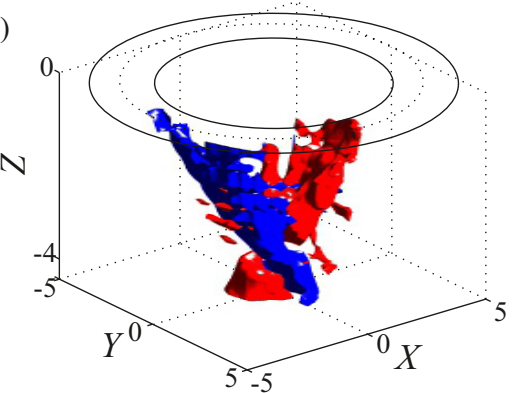

FIG. 11. Isosurface of the instantaneous vertical vorticity field $\xi_{z} / N= \pm 0.03$ measured at phase $\omega_{0} t=\pi$ of the 23rd oscillation period, reconstructed from the volume PIV measurements for (a) internal waves and (b) inertia-gravity waves. The value 0.03 has been chosen at $60 \%$ of the vorticity maximum, 0.05 , which is the same for both wave types as seen in Figs. 10(c) and 10(e). Red and blue colors indicate positive and negative vorticity, respectively, and their sign changes every half period. The horizontal section of the torus at $Z=0$ is shown with black curves. See Expts. 1.2 and 2.2 from Table I.

to be $150 \mathrm{~cm}$, which corresponds to the size of the torus, and is thus the maximum size the vortex structure may have (see Fig. 11).

\section{B. Time-frequency representation, higher harmonics, and resonance}

In order to determine the wave spectrum generated by the torus in a stratified and rotating fluid as well as its time evolution, we use the expression of [39] for the time-frequency spectrum, which for the density disturbance $\rho$ gives

$$
Q_{\rho}(t, \omega)=\left|\int_{-\infty}^{+\infty} \rho(\tau) e^{-i \omega \tau} h(t-\tau) d \tau\right|^{2},
$$

with $h(t)$ a smoothing Hamming window. Figure 12 shows the evolution of the spectrum over 21 oscillation periods, with the time normalized as $T=\left(\omega_{0} t\right) / 2 \pi$. The data have been obtained from the conductivity probe placed near the focal region. Though taken at a single point, they have been acquired at a much higher frequency and are better resolved in time and amplitude than the PIV data (not shown).

For internal waves [Figs. 12(a) and 12(b)] there is a gradual increase in the presence of subharmonics with time, while higher harmonics with frequencies that are integer multiples of the forcing frequency $\omega_{0}$ become more energetic. The permanent presence of both sub- and higher harmonics almost from the beginning of the oscillations suggests strongly nonlinear effects even for low oscillation amplitudes. Increasing the oscillation amplitude [see Fig. 12(b)] also increases the amplitudes of these nonlinear effects, in addition to slight variation with time. Most of the energy is then transferred into higher harmonics.

Almost the same tendency can be observed for inertia-gravity waves [see Figs. 12(c) and 12(d)]. The subharmonics are however less present for small oscillation amplitudes, and the amplitudes of the fundamental wave of frequency $\omega_{0}$ and higher harmonics of frequencies $n \omega_{0}$ are more pronounced. Cross sections taken at $T=12.5$ in Figs. 12(a) and 12(b) and Figs. 12(c) and 12(d) are represented in Figs. 13(a) and 13(b), respectively. For larger amplitudes, most frequencies continue to exist, likely due to resonances. The sub- and higher harmonics become relatively more important compared to the fundamental wave, and small scales have more energy, in particular for inertia-gravity waves.

These higher harmonics are generated due to nonlinear effects in regions where the wave amplitude is high, such as in the boundary layer of the oscillating object $[25,26]$ and in the focal 


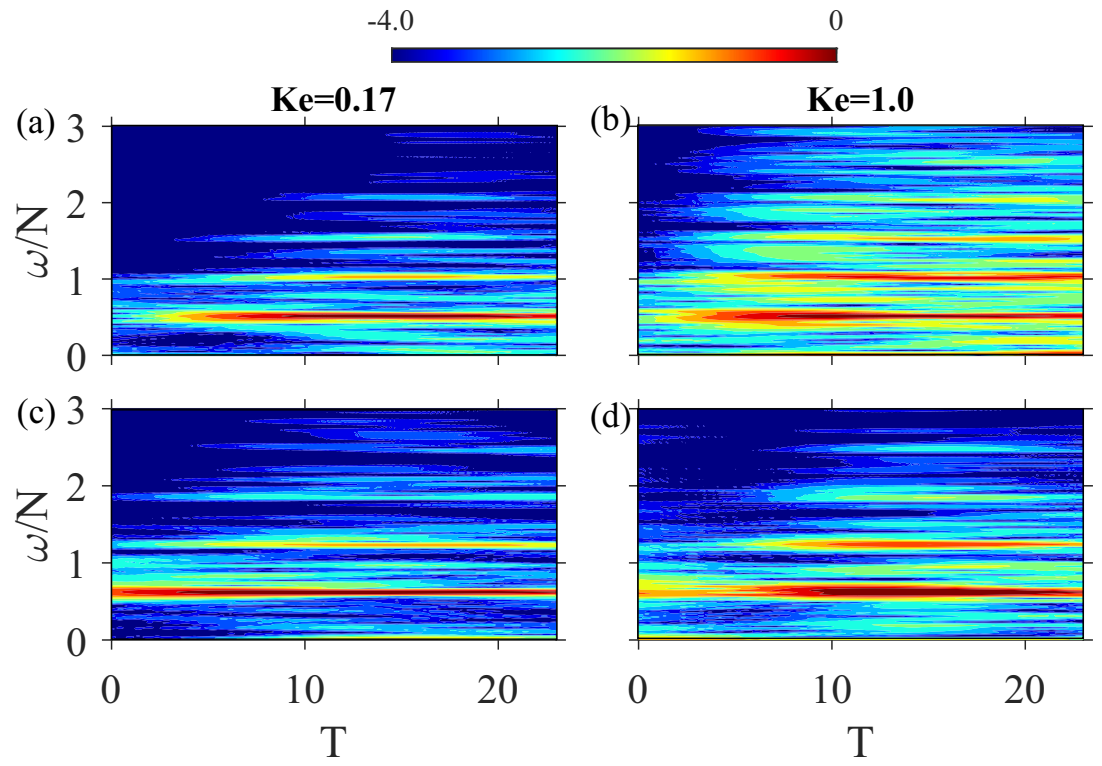

FIG. 12. Time-frequency spectra $\log _{10}\left[Q_{\rho}(t, \omega) / Q_{0}\right]$ obtained from probe data, with $Q_{0}=$ $\max _{t}\left[Q_{\rho}\left(t, \omega_{0}\right)\right]$, for (a), (b) internal waves and (c), (d) inertia-gravity waves at (a), (c) $\mathrm{Ke}=0.17$ and (b), (d) $\mathrm{Ke}=1.0$.

zone, and have higher amplitudes for higher oscillation amplitudes. A cascade of higher frequencies was shown in the numerical simulations of the tidal motion over a flat-topped ocean ridge by Korobov and Lamb [40], similar to the profiles shown here in Figs. 13(a) and 13(b).

Teoh et al. [41] and Javam et al. [42] considered the interaction of internal wave beams generated with two horizontal paddles, and later also Smith and Crockett [43] using two wave generators made of superposed acrylic plates. In both cases, the interaction between the waves was nonlinear but nonresonant. This purely two-dimensional forcing resulted also in the radiation of standing higher evanescent modes $[42,44]$ with a density fluctuation spectrum similar to that in Fig. 13(a) with higher harmonics generation (see Fig. 12 for $\omega / N=0.51$ in [41]). Though their Stokes number was
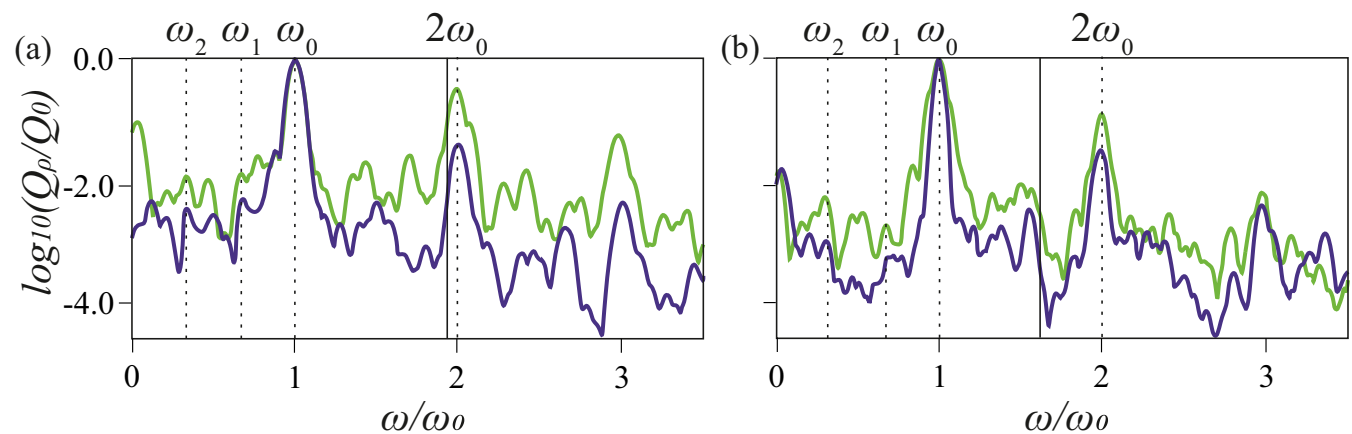

FIG. 13. Typical spectra taken at $T=12.5$ for (a) internal waves and (b) inertia-gravity waves, with $\mathrm{Ke}=$ 0.17 (purple line) and $\mathrm{Ke}=1.0$ (green line). The solid vertical line indicates the buoyancy frequency $N$ and the dashed lines the fundamental frequency $\omega_{0}$, the second harmonic frequency $2 \omega_{0}$, and the subharmonic frequencies $\omega_{1}$ and $\omega_{2}$. 

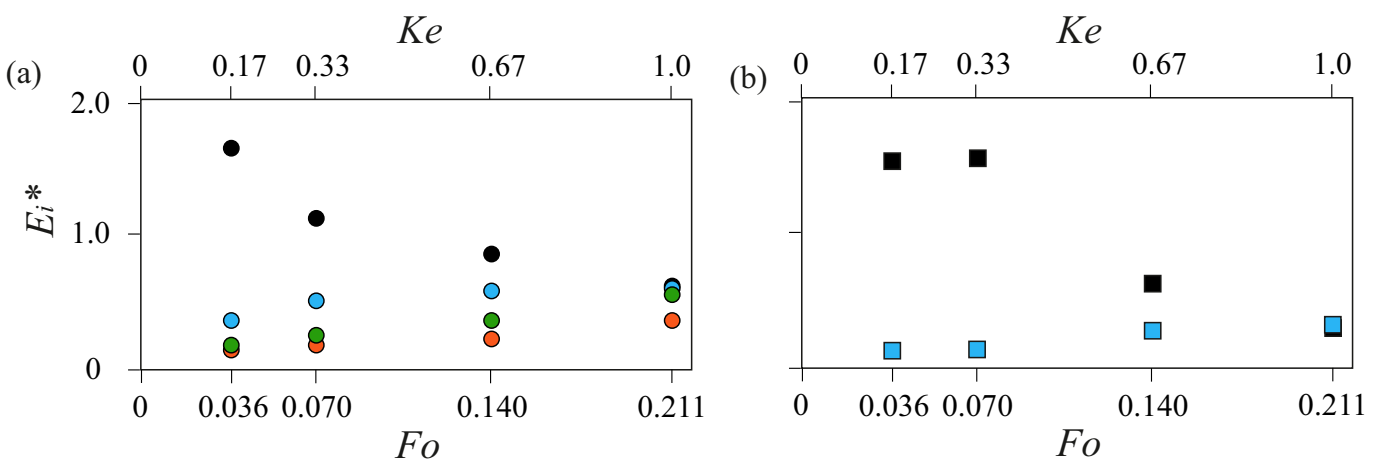

FIG. 14. Maximum kinetic energy density $E^{*}$ of the fundamental wave of frequency $\omega_{0}$ (black symbols), second harmonic wave of frequency $2 \omega_{0}$ (blue symbols), and two subharmonic waves of frequencies $\omega_{1}$ and $\omega_{2}$ (red and green symbols, respectively), as functions of the Keulegan-Carpenter number Ke and the focusing number Fo, for (a) internal waves and (b) inertia-gravity waves, as obtained from (three-dimensional) PIV measurements. As discussed in Sec. IV A, the second harmonic component in (b) is only made of noise for $\mathrm{Ke}=0.17$ and 0.33 and hence conveys no energy. Its increase in energy for $\mathrm{Ke}=0.67$ and 1.0 is due to wave generation by the turbulence in the boundary layer of the torus, the waves having a very noisy structure in contrast to the cases shown in (a).

huge, $\mathrm{O}(13000)$, and $\mathrm{Ke}=0.14$, the modes in between were absent or very weak. The difference comes most likely from the focusing in the present experiments. Higher harmonics were also observed in the small-scale experiments of [15]. Their amplitude increases with time. This energy transfer towards higher frequencies is well known for the nonlinear interaction of, e.g., incident and reflected waves near sloping boundaries.

Figures 14(a) and 14(b) show for internal waves and inertia-gravity waves, respectively, the maximum kinetic energy density $E^{*}=1 / 2\left(U^{2}+V^{2}+W^{2}\right)$ of the different harmonics over the entire volume below the torus, determined from the volume PIV data, as a function of the oscillation amplitude represented by Ke (or Fo). The systematic saturation of the energy of the fundamental wave with increasing oscillation amplitude, akin to the saturation phenomenon reported for an oscillating sphere in $[13,14]$, coincides with the increase of the energy of the higher harmonics. Though there is more noise in the PIV data of the inertia-gravity waves, the same tendency is observed [see Fig. 14(b)]. While the fundamental wave is by far dominant in the linear regime, $\mathrm{Ke}<0.33$, at larger amplitudes subharmonics become non-negligible, until for $\mathrm{Ke} \approx 1$ all waves are of a comparable order. As mentioned above and shown in Fig. 4, the dominant presence of subharmonics changes the flow character, in contrast to the moderate-Stokes-number flows studied in $[17,18]$.

\section{Resonance}

Triadic resonant interaction of internal and inertial waves has been exhibited by [45,46], respectively, while, for the waves generated by an oscillating torus, a similar resonance was found by [18] for moderate Stokes numbers and unimodal waves. Here we consider large Stokes numbers and bimodal waves. Though this motion is three dimensional, we follow $[45,46]$ and consider the motion in the vertical symmetry plane, i.e., the $X Z$ plane. In this plane, two secondary waves of frequencies $\omega_{1}$ and $\omega_{2}$ and respective wave vectors $\mathbf{k}_{1}$ and $\mathbf{k}_{2}$ form a resonant triad together with a primary wave of frequency $\omega_{0}$ and wave vector $\mathbf{k}_{0}$ when they satisfy the resonance conditions

$$
\omega_{0}=\omega_{1}+\omega_{2}, \quad \mathbf{k}_{0}=\mathbf{k}_{1}+\mathbf{k}_{2},
$$




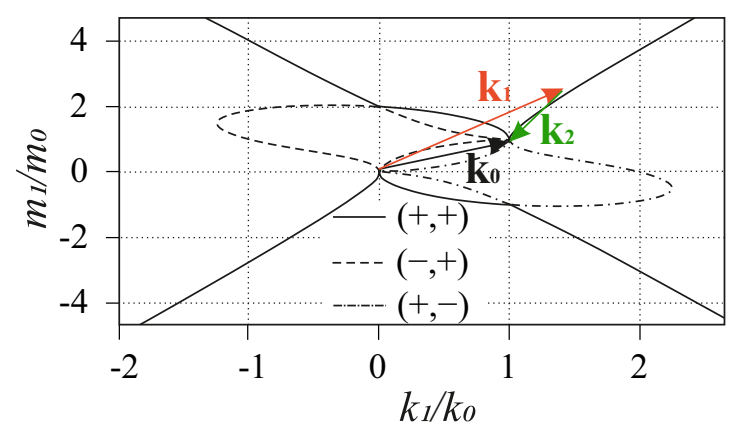

FIG. 15. Resonance diagram associated to the triadic wave components for internal waves, showing the solutions of Eq. (12) for $\omega_{0} / N=0.51$ together with the experimental wave vectors. The solid lines correspond to unstable interactions and the dashed and dash-dotted lines to stable interactions. See Expt. 1.1 from Table I.

together with, for each wave, the dispersion relation between the frequency $\omega_{i}$ and the wave vector $\mathbf{k}_{i}=\left(k_{i}, l_{i}=0, m_{i}\right)$, namely

$$
\omega_{i}=s_{i} \sqrt{\frac{N^{2} k_{i}^{2}+f^{2} m_{i}^{2}}{k_{i}^{2}+m_{i}^{2}}},
$$

where $s_{i}= \pm 1$ defines the sign of the wave. Without loss of generality, we take $s_{0}=1$. As shown in [47], only the "sum" interactions with signs $\left(s_{1}, s_{2}\right)=(+,+)$ have growth rates with a positive real part, while the "difference" interactions with signs $(+,-)$ and $(-,+)$ have growth rates with a zero real part in an inviscid fluid and a negative real part in a viscous fluid; the interactions with signs $(-,-)$ are excluded from the outset. Accordingly, only subharmonic waves of frequencies $f<\omega_{1,2}<\omega_{0}$ can form an unstable triad with the fundamental wave of frequency $\omega_{0}$ through "sum" interactions.

For internal waves $[5,45,48]$, the combination of Eqs. (10) and (11) leads to

$$
\begin{aligned}
1= & s_{1} \frac{\left|k_{1} / k_{0}\right|}{\sqrt{\left(k_{1} / k_{0}\right)^{2}\left(\omega_{0} / N\right)^{2}+\left(m_{1} / m_{0}\right)^{2}\left[1-\left(\omega_{0} / N\right)^{2}\right]}} \\
& +s_{2} \frac{\left|1-\left(k_{1} / k_{0}\right)\right|}{\sqrt{\left[1-\left(k_{1} / k_{0}\right)\right]^{2}\left(\omega_{0} / N\right)^{2}+\left[1-\left(m_{1} / m_{0}\right)\right]^{2}\left[1-\left(\omega_{0} / N\right)^{2}\right]}},
\end{aligned}
$$

yielding for $\omega_{0} / N=0.51$ the resonance diagram in Fig. 15 .

Resonant triads were observed for unimodal waves in [18], with their amplitude increasing with the oscillation amplitude of the forcing. For the bimodal waves observed here, the direction of propagation of each wave has been deduced from the plots in Fig. 16, showing the phase of the waves as obtained after applying the Hilbert transform [38] to the experimental fields. The comparison of the experimental wave vectors with the solution of Eq. (12) is shown in Fig. 15. The resonance, at $\omega_{1} / \omega_{0}=0.67$ and $\omega_{2} / \omega_{0}=0.33$, is close to that found in [45], suggesting that it is robust and recurrent in three dimensions, for unimodal waves as well as for bimodal waves. However, Ref. [45] showed these three resonant frequencies against a quiet background, as did [18] but with $\omega_{1}$ and $\omega_{2}$ having less energy due to three-dimensional effects. Here, the amplitudes of $\omega_{1}$ and $\omega_{2}$ are even smaller and are accompanied by a number of other frequencies, among which other triadic combinations are possible (e.g., $\omega / \omega_{0}=0.88$ and $\omega / \omega_{0}=0.12$ ) (see Fig. 13). For the interaction of two internal wave beams of frequencies $\omega_{1}$ and $\omega_{2}$ the theory in [49] and the experiments in $[50,51]$ indicate that the interaction region contains components not only of the original frequencies $\left(\omega_{1}\right.$ and $\left.\omega_{2}\right)$, but also at the difference frequency $\left|\omega_{1}-\omega_{2}\right|$ and combination frequencies $2 \omega_{1}-\omega_{2}$, $2 \omega_{2}-\omega_{1}, 3 \omega_{1}-\omega_{2}$, etc. Above we have only verified that the wave vectors obtained from the 


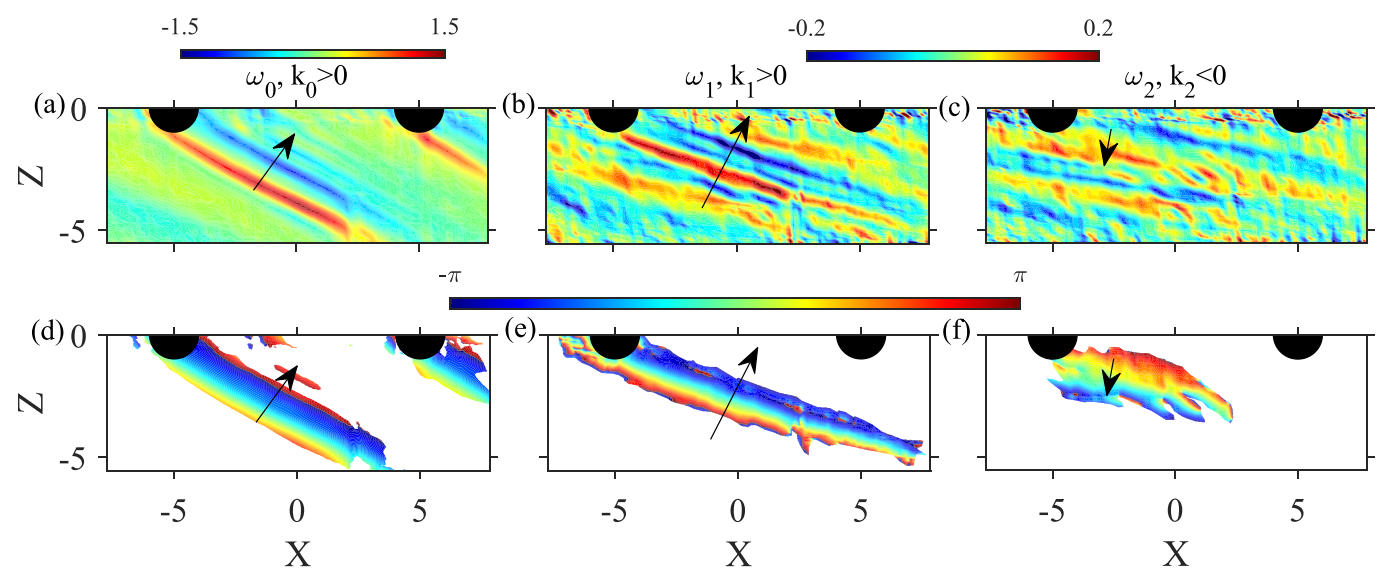

FIG. 16. Instantaneous horizontal velocity fields from PIV data (for $\mathrm{Ke}=0.17$ ) filtered in time and space around (a) $\omega_{0}$ and $k_{0}>0$, (b) $\omega_{1}$ and $k_{1}>0$, and (c) $\omega_{2}$ and $k_{2}<0$, and associated phase plots in (d), (e), and (f), respectively, for the triadic wave components of internal waves.

PIV measurements correspond to a single combination of frequencies $\omega_{1}$ and $\omega_{2}$. The resolution of the PIV data was however not sufficient to verify the dispersion relation of resonant triads for the combination of these other frequencies in Fig. 13.

Ghaemsaidi and Mathur [52] considered, in addition to two-dimensional resonant interactions, also three-dimensional instabilities as a function of Ke and the angle of wave propagation. The threedimensional effects become relevant for $\mathrm{Ke}>1$. Though we notice here the presence of a range of waves with multiple interactions, they appear to satisfy the two-dimensional dispersion relation represented graphically in Fig. 15, unlike the three-dimensional effect of focusing, suggesting a dominant two-dimensional dynamics.

In the case of inertia-gravity waves [53-55], Eq. (12) for the resonance locus becomes

$$
\begin{aligned}
\frac{\omega_{0}}{N}= & s_{1} \sqrt{\frac{\left[\left(\omega_{0} / N\right)^{2}-(f / N)^{2}\right]\left(k_{1} / k_{0}\right)^{2}+(f / N)^{2}\left[1-\left(\omega_{0} / N\right)^{2}\right]\left(m_{1} / m_{0}\right)^{2}}{\left[\left(\omega_{0} / N\right)^{2}-(f / N)^{2}\right]\left(k_{1} / k_{0}\right)^{2}+\left[1-\left(\omega_{0} / N\right)^{2}\right]\left(m_{1} / m_{0}\right)^{2}}} \\
& +s_{2} \sqrt{\frac{\left[\left(\omega_{0} / N\right)^{2}-(f / N)^{2}\right]\left[1-\left(k_{1} / k_{0}\right)\right]^{2}+(f / N)^{2}\left[1-\left(\omega_{0} / N\right)^{2}\right]\left[1-\left(m_{1} / m_{0}\right)\right]^{2}}{\left[\left(\omega_{0} / N\right)^{2}-(f / N)^{2}\right]\left[1-\left(k_{1} / k_{0}\right)\right]^{2}+\left[1-\left(\omega_{0} / N\right)^{2}\right]\left[1-\left(m_{1} / m_{0}\right)\right]^{2}}},
\end{aligned}
$$

yielding for $\theta=59^{\circ}$ the resonance diagrams in Fig. 17 as the Prandtl ratio $f / N$ varies from 0.1 to 0.4 . As these diagrams show, owing to the condition $f<\omega_{1,2}<\omega_{0}$, unstable "sum" triads can only exist provided that $\omega_{0}=\omega_{1}+\omega_{2}>2 f$, hence for $f / N<\omega_{0} /(2 N)$; that is, at given $\theta$ for $f / N<\cos \theta / \sqrt{3+\cos ^{2} \theta}$, here $f / N<0.28$, and at given $f / N$ for $\omega_{0} / N>2 f / N$, here $\omega_{0} / N>$ 0.8. This criterion is specific to triadic resonance. Using Lagrangian-mean theory Dong et al. [56] also observed a value of $f / N$ above which no resonant triad can occur, in their setup $f / N>0.4$. Either approach implies that there should be no unstable triad in the present experiments for which $f / N=0.4$. Even though subharmonics are present in Fig. 12(c), there are indeed no associated wave vectors that can satisfy the dispersion relation (11) and resonant conditions (10) simultaneously.

Resonant inertia-gravity waves have been considered in [53-56], and experimentally for twodimensional forcing by [54]. Maurer et al. [57] considered axisymmetric forcing allowing for geometric focusing, using a set of circular concentric pistons that oscillated vertically in a rotating and stratified basin for Prandtl ratio $f / N=0.2$. Though resonance was observed in the focal region on the common axes of the cylindrical pistons, it was not further detailed since the primary purpose of these experiments was the properties of the wave maker. 

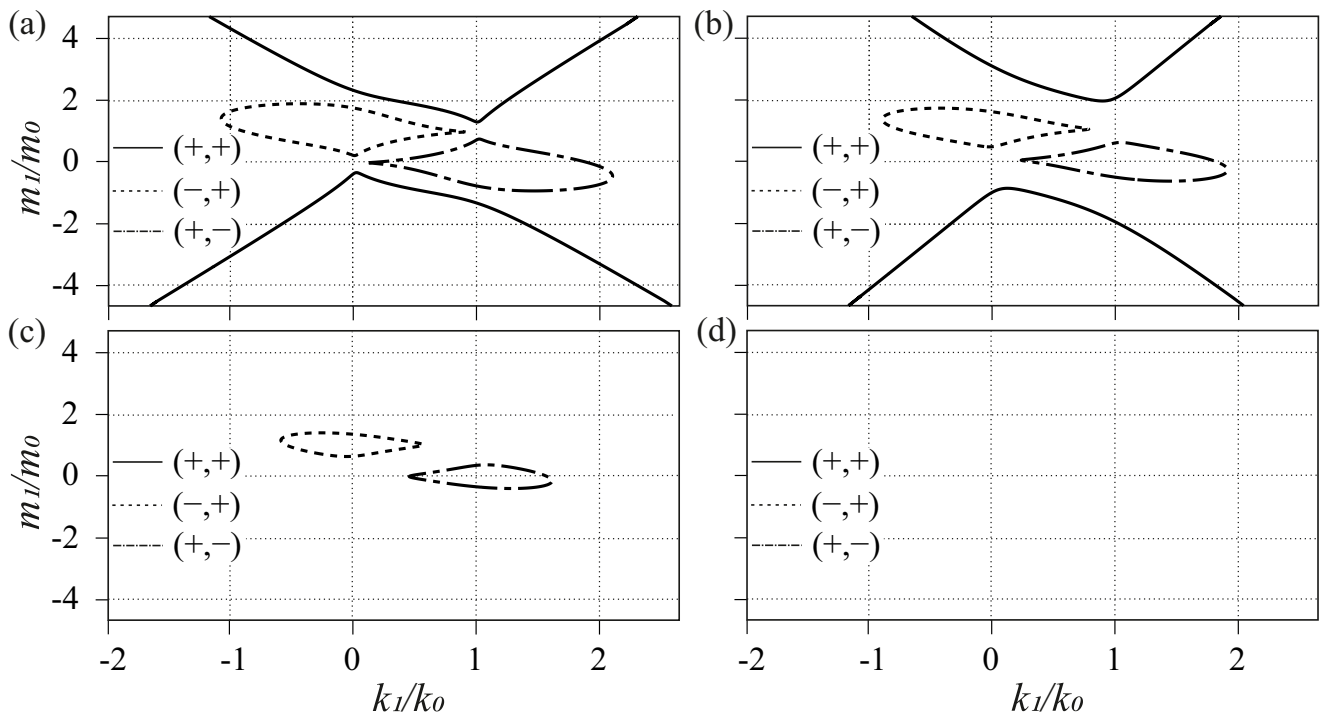

FIG. 17. Resonance diagrams of inertia-gravity waves, showing the solutions of Eq. (13) for $(+,+)$ interactions (solid lines), $(-,+)$ interactions (dashed lines), and $(+,-)$ interactions (dash-dotted lines) at $\theta=59^{\circ}$ for (a) $f / N=0.1$, (b) $f / N=0.2$, (c) $f / N=0.3$, and (d) $f / N=0.4$.

\section{CONCLUSIONS AND DISCUSSION}

In this study we have considered the wave focusing caused by the oscillations of a torus in a stratified fluid with or without background rotation. The results show bimodal waves with four focal zones where the velocity amplifies to a maximum. Nonlinear flow features such as the generation of higher harmonics in the boundary layer of the torus, together with triadic resonance yielding subharmonics in the focal region, are observed. Generally, compared to the moderate-Stokes-number flows studied in $[17,18]$, the wave rays are thicker and have a reduced maximum amplitude for large Stokes numbers St, and this effect becomes even more pronounced for larger forcing amplitude, i.e., for larger Keulegan-Carpenter numbers Ke. Given the absence of other effects, this must be due to the diffusing effect of small-scale motions, here little affected by viscosity.

For small forcing amplitudes, $\mathrm{Ke} \leqslant 0.17$, the overall structure of the focused waves is preserved and compares well with the linear viscous theory of [27], of which an amended version has been presented in Sec. II. The triadic resonance that is observed for these bimodal waves compares well to the observations of $[17,18]$ for moderate Stokes numbers and unimodal waves. These wave triads are similar to the triads observed by $[45,46]$ in flows with a two-dimensional wave forcing.

For larger oscillation amplitudes, $\mathrm{Ke}>0.17$, the waves become fully nonlinear with oscillatory motions at small scales as shown in Figs. 4(1) and 4(o). For these large Stokes numbers, the Richardson number appears to remain constant due to the generation of small-scale turbulence in the boundary layer and the relatively important generation of higher harmonics compared to the fundamental wave. As a consequence, the waves do not overturn and the flow represents a state of wave turbulence.

The motion diffuses over a larger area and the wave rays have a smaller amplitude. In the boundary layer and the focal region, different harmonics are generated with a comparable amplitude and relatively less energy for the fundamental wave. Thus, for large oscillation amplitudes, the amplitude of the fundamental decreases, while the amplitude of higher harmonics slightly increases (see Figs. 14). This flow picture is in sharp contrast with its moderate-Stokes-number counterpart (see [18]), for which an increase in forcing amplitude results in an increase of only the fundamental wave, with resonance and overturning occurring in the focal region. 
The evolution of inertia-gravity waves has been considered for one forcing frequency and, except for the presence of resonance, is comparable to the evolution for small forcing amplitudes. For $\mathrm{Ke}=0.17$ there is no resonance, since the forcing frequency is smaller than twice the Coriolis parameter, a frequency range for which no triadic interaction can take place. For higher-amplitude forcing the energy spectra nevertheless suggest the presence of resonance. For Ke approaching 1 the PIV data in Fig. 14(b) suggest a similar trend as observed for internal waves, with higher harmonics becoming as important as the fundamental wave in Fig. 14(a). But the more accurate probe data for the spectra show that less energy is distributed into the higher harmonics [Fig. 12(c)], which is probably due to this difference in resonance.

In a forthcoming paper, the flow variations with the Stokes number, and also the contributions of streaming and Stokes drift to the mean flow, will be considered. Though some observations were made in the present investigation, the overwhelming diffusive effect for large Stokes numbers, in particular for large amplitudes, led to poorly conclusive results.

All the preceding results have been obtained in an axisymmetric configuration, the circular ring, yielding optimal focusing. Departures from axisymmetry can be of two kinds. First, the ring can no longer be circular. Bühler and Muller [22] considered Gaussian topography in the shape of an elliptic ring, theoretically, and Shmakova et al. [15] a spheroid of horizontal axis, experimentally. In both cases, the focal points on the axis of symmetry for the circular case degenerate into curves. The wave energy is spread over larger focal regions, and focusing remains present but is less efficient. In [22], the amplification rate between the wave amplitudes at the foci and at generation was seen to be divided by a factor of about 1.5 , compared with the circular case. Second, the ring can still be circular but undergo azimuthal variations. Grisouard and Bühler [23] considered horseshoe-shaped circular Gaussian topography, and Voisin [27] the same topography together with half and quarter tori with various orientations with respect to the barotropic oscillation. Both investigations were theoretical. Their conclusions were the same as for a noncircular ring: focusing remains present but is less efficient.

In the oceans the Stokes number is much larger than in the present experiments, whereas the Keulegan-Carpenter number is generally much smaller. Consider a typical seamount with a height of $320 \mathrm{~m}$ and a radius of $1600 \mathrm{~m}$ over which flows the $M_{2}$ tide with a frequency of $2 \times 10^{-4} \mathrm{~s}^{-1}$ and a velocity amplitude of $1.4 \times 10^{-2} \mathrm{~m} / \mathrm{s}$ (see the seamount statistics of [58,59]), and a turbulent kinematic viscosity of $10^{-5} \mathrm{~m}^{2} / \mathrm{s}$ that is used for the $M_{2}$ tide by [60]. This yields St of the order of $5 \times 10^{7}$ and $\mathrm{Ke}$ of the order of $5 \times 10^{-2}$. Smaller seamounts in height (and width) may have Ke larger than 0.17. Mixing due to resonance will be generated by this larger-size topography (smaller Ke), whereas the smaller-scale structures (larger Ke) will generate wave turbulence without overturning motions and therefore hardly contribute to the mixing.

To conclude, for these higher Stokes numbers, the wave pattern is more diffuse and more energy is transferred into higher harmonics, in particular when the forcing amplitude is in the nonlinear regime. This change in the flow structure is very different from its moderate-Stokes-number counterpart.

\section{ACKNOWLEDGMENTS}

The authors acknowledge the help of S. Viboud and T. Valran for setting up the experiments at the Coriolis platform. N.S. acknowledges support by Université Grenoble Alpes (UGA), which funded her Ph.D. thesis (2016) in the context of which these experiments have been conducted, integration project III.22.4.3 of the Siberian Branch of the Russian Academy of Sciences, and the Metchnikov fellowship 2019 from the French Embassy in the Russian Federation, which allowed for further processing of the data. This work has been supported by a grant from LabEx OSUG@2020 (Investissements d'avenir ANR10 LABX56) and Fondation Simone et Cino Del Duca. The referees are thanked for helpful suggestions and comments. 
[1] C. Garrett, Internal tides and ocean mixing, Science 301, 1858 (2003).

[2] R. Ferrari and C. Wunsch, Ocean circulation kinetic energy: reservoirs, sources, and sinks, Annu. Rev. Fluid Mech. 41, 253 (2009).

[3] R. Ferrari, What goes down must come up, Nature (London) 513, 179 (2014).

[4] L. Cheng, J. Abraham, Z. Hausfather, and K. E. Trenberth, How fast are the oceans warming?, Science 363, 128 (2019).

[5] T. Dauxois, S. Joubaud, P. Odier, and A. Venaille, Instabilities of internal gravity wave beams, Annu. Rev. Fluid Mech. 50, 131 (2018).

[6] H. P. Zhang, B. King, and H. L. Swinney, Experimental study of internal gravity waves generated by supercritical topography, Phys. Fluids 19, 096602 (2007).

[7] E. V. Ermanyuk and N. V. Gavrilov, On internal waves generated by large-amplitude circular and rectilinear oscillations of a circular cylinder in a uniformly stratified fluid, J. Fluid Mech. 613, 329 (2008).

[8] L. Gostiaux and T. Dauxois, Laboratory experiments on the generation of internal tidal beams over steep slopes, Phys. Fluids 19, 028102 (2007).

[9] H. P. Zhang, B. King, and H. L. Swinney, Resonant Generation of Internal Waves on a Model Continental Slope, Phys. Rev. Lett. 100, 244504 (2008).

[10] T. Peacock, P. Echeverri, and N. J. Balmforth, An experimental investigation of internal tide generation by two-dimensional topography, J. Phys. Oceanogr. 38, 235 (2008).

[11] P. Echeverri, M. R. Flynn, K. B. Winters, and T. Peacock, Low-mode internal tide generation by topography: an experimental and numerical investigation, J. Fluid Mech. 636, 91 (2009).

[12] B. King, H. P. Zhang, and H. L. Swinney, Tidal flow over three-dimensional topography in a stratified fluid, Phys. Fluids 21, 116601 (2009).

[13] B. Voisin, E. V. Ermanyuk, and J.-B. Flór, Internal wave generation by oscillation of a sphere, with application to internal tides, J. Fluid Mech. 666, 308 (2011).

[14] E. V. Ermanyuk, J.-B. Flór, and B. Voisin, Spatial structure of first and higher harmonic internal waves from a horizontally oscillating sphere, J. Fluid Mech. 671, 364 (2011).

[15] N. Shmakova, E. Ermanyuk, and J.-B. Flór, Generation of higher harmonic internal waves by oscillating spheroids, Phys. Rev. Fluids 2, 114801 (2017).

[16] B. King, H. P. Zhang, and H. L. Swinney, Tidal flow over three-dimensional topography generates outof-forcing-plane harmonics, Geophys. Res. Lett. 37, L14606 (2010).

[17] E. V. Ermanyuk, N. D. Shmakova, and J.-B. Flór, Internal wave focusing by a horizontally oscillating torus, J. Fluid Mech. 813, 695 (2017).

[18] N. D. Shmakova and J.-B. Flór, Nonlinear aspects of focusing internal waves, J. Fluid Mech. 862, R4 (2019).

[19] M. C. Buijsman, J. M. Klymak, S. Legg, M. H. Alford, D. Farmer, J. A. MacKinnon, J. D. Nash, J.-H. Park, A. Pickering, and H. Simmons, Three-dimensional double-ridge internal tide resonance in Luzon Strait, J. Phys. Oceanogr. 44, 850 (2014).

[20] A. Peliz, B. Le Cann, and C. Mohn, Circulation and mixing in a deep submerged crater: Tore seamount, Geophys. Res. Abstr. 11, EGU2009-7567-1 (2009).

[21] V. Vlasenko, N. Stashchuk, M. E. Inall, M. Porter, and D. Aleynik, Focusing of baroclinic tidal energy in a canyon, J. Geophys. Res. Oceans 121, 2824 (2016).

[22] O. Bühler and C. J. Muller, Instability and focusing of internal tides in the deep ocean, J. Fluid Mech. 588, 1 (2007).

[23] N. Grisouard and O. Bühler, Forcing of oceanic mean flows by dissipating internal tides, J. Fluid Mech. 708, 250 (2012).

[24] M. Duran-Matute, J.-B. Flór, F. S. Godeferd, and C. Jause-Labert, Turbulence and columnar vortex formation through inertial-wave focusing, Phys. Rev. E 87, 041001(R) (2013).

[25] T. H. Bell, Lee waves in stratified flows with simple harmonic time dependence, J. Fluid Mech. 67, 705 (1975).

[26] T. H. Bell, Topographically generated internal waves in the open ocean, J. Geophys. Res. 80, 320 (1975). 
[27] B. Voisin, Internal wave focusing by annular forcing, in 8th International Symposium on Stratified Flows (San Diego, 2016).

[28] B. Voisin, Near-field internal wave beams in two dimensions, J. Fluid Mech. 900, A3 (2020).

[29] J. N. Newman, The motions of a floating slender torus, J. Fluid Mech. 83, 721 (1977).

[30] D. G. Hurley, The generation of internal waves by vibrating elliptic cylinders. Part 1. Inviscid solution, J. Fluid Mech. 351, 105 (1997).

[31] D. G. Hurley and G. Keady, The generation of internal waves by vibrating elliptic cylinders. Part 2. Approximate viscous solution, J. Fluid Mech. 351, 119 (1997).

[32] B. Voisin, Limit states of internal wave beams, J. Fluid Mech. 496, 243 (2003).

[33] L. M. Brekhovskikh and V. Goncharov, Mechanics of Continua and Wave Dynamics, 2nd ed. (Springer, Berlin, 1994).

[34] http://www.legi.cnrs.fr/web/spip.php?article763.

[35] A. M. J. Davis and S. G. Llewellyn Smith, Tangential oscillations of a circular disk in a viscous stratified fluid, J. Fluid Mech. 656, 342 (2010).

[36] See Supplemental Material at http://link.aps.org/supplemental/10.1103/PhysRevFluids.6.114804 for (I) a two-dimensional view of internal waves during the 17th oscillation period in the vertical plane $Y=0$ for the horizontal velocity $U$ (left column), vertical velocity $W$ (middle column), and transverse vorticity $\xi_{y} / N$ with velocity vectors (right column), and (II) a three-dimensional view of the vertical vorticity $\xi_{z} / N$ of internal waves (left) and inertia-gravity waves (right) during the 23rd oscillation period, with isosurfaces of negative vorticity in blue and positive vorticity in red.

[37] Ž. Nosan, F. Burmann, P. A. Davidson, and J. Noir, Evanescent inertial waves, J. Fluid Mech. 918, R2 (2021).

[38] M. J. Mercier, N. B. Garnier, and T. Dauxois, Reflection and diffraction of internal waves analyzed with the Hilbert transform., Phys. Fluids 20, 086601 (2008).

[39] P. Flandrin, Time-Frequency/Time-Scale Analysis (Academic, New York, 1999).

[40] A. S. Korobov and K. G. Lamb, Interharmonics in internal gravity waves generated by tide-topography interaction, J. Fluid Mech. 611, 61 (2008).

[41] S. G. Teoh, G. N. Ivey, and J. Imberger, Laboratory study of the interaction between two internal wave rays, J. Fluid Mech. 336, 91 (1997).

[42] A. Javam, J. Imberger, and S. W. Armfield, Numerical study of internal wave-wave interactions in a stratified fluid, J. Fluid Mech. 415, 65 (2000).

[43] S. Smith and J. Crockett, Experiments on nonlinear harmonic wave generation from colliding internal wave beams, Exp. Therm. Fluid Sci. 54, 93 (2014).

[44] A. Javam, S. G. Teoh, J. Imberger, and G. N. Ivey, Two intersecting internal wave rays: A comparison between numerical and laboratory results, in Physical Processes in Lakes and Oceans, edited by $\mathrm{J}$. Imberger (American Geophysical Union, Washington, DC, 1998), pp. 241-250.

[45] B. Bourget, T. Dauxois, S. Joubaud, and P. Odier, Experimental study of parametric subharmonic instability for internal plane waves, J. Fluid Mech. 723, 1 (2013).

[46] G. Bordes, F. Moisy, T. Dauxois, and P.-P. Cortet, Experimental evidence of a triadic resonance of plane inertial waves in a rotating fluid, Phys. Fluids 24, 014105 (2012).

[47] K. Hasselmann, A criterion for nonlinear wave stability, J. Fluid Mech. 30, 737 (1967).

[48] C. Staquet and J. Sommeria, Internal gravity waves: from instabilities to turbulence, Annu. Rev. Fluid Mech. 34, 559 (2002).

[49] A. V. Kistovich and Y. D. Chashechkin, Nonlinear interaction of two-dimensional packets of monochromatic internal waves, Izv. Atmos. Ocean. Phys. 27, 946 (1991).

[50] Y. D. Chashechkin and V. I. Neklyudov, Nonlinear interaction of bundles of short two-dimensional monochromatic internal waves in an exponentially stratified liquid, Trans. (Dokl.) Acad. Sci. USSR Earth Sci. Sect. 311, 235 (1990).

[51] A. V. Kistovich, V. I. Neklyudov, and Y. D. Chashechkin, Nonlinear effects in the generation and interaction of packets of harmonic internal waves, Fluid Mech. Res. 21(5), 117 (1992).

[52] S. J. Ghaemsaidi and M. Mathur, Three-dimensional small-scale instabilities of plane internal gravity waves, J. Fluid Mech. 863(5), 702 (2019). 
[53] C. H. McComas and F. P. Bretherton, Resonant interaction of oceanic internal waves, J. Geophys. Res. 82, 1397 (1977).

[54] P. Maurer, S. Joubaud, and P. Odier, Generation and stability of inertia-gravity waves, J. Fluid Mech. 808, 539 (2016).

[55] O. Richet, J.-M. Chomaz, and C. Muller, Internal tide dissipation at topography: Triadic resonant instability equatorward and evanescent waves poleward of the critical latitude, J. Geophys. Res. Oceans 123, 6136 (2018).

[56] W. Dong, O. Bühler, and K. S. Smith, Mean flows induced by inertia-gravity waves in a vertically confined domain, J. Fluid Mech. 890, A6 (2020).

[57] P. Maurer, S. J. Ghaemsaidi, S. Joubaud, T. Peacock, and P. Odier, An axisymmetric inertia-gravity wave generator, Exp. Fluids 58, 143 (2017).

[58] T. H. Jordan, H. W. Menard, and D. K. Smith, Density and size distribution of seamounts in the eastern Pacific inferred from wide-beam sounding data, J. Geophys. Res. 88, 10508 (1983).

[59] D. K. Smith and T. H. Jordan, Seamount statistics in the Pacific Ocean, J. Geophys. Res. 93, 2899 (1988).

[60] E. Di Lorenzo, W. R. Young, and S. Llewellyn Smith, Numerical and analytical estimates of $M_{2}$ tidal conversion at steep oceanic ridges, J. Phys. Oceanogr. 36, 1072 (2006). 